\title{
Unit Distances and Diameters in Euclidean Spaces
}

\author{
Konrad J. Swanepoel
}

Received: 2 July 2007 / Revised: 14 March 2008 / Accepted: 6 April 2008 / Published online: 8 May 2008 (C) Springer Science+Business Media, LLC 2008

\begin{abstract}
We show that the maximum number of unit distances or of diameters in a set of $n$ points in $d$-dimensional Euclidean space is attained only by specific types of Lenz constructions, for all $d \geq 4$ and $n$ sufficiently large depending on $d$. As a corollary, we determine the exact maximum number of unit distances for all even $d \geq 6$ and the exact maximum number of diameters for all $d \geq 4$ and all $n$ sufficiently large depending on $d$.
\end{abstract}

Keywords Erdős problem · Combinatorial geometry $\cdot$ Unit distance problem $\cdot$ Lenz construction $\cdot$ Diameters $\cdot$ Erdôs-Simonovits stability theorem $\cdot$ Erdôs-Stone theorem

\section{Introduction}

\subsection{Unit Distances}

For a finite subset $S$ of Euclidean $d$-space $\mathbb{R}^{d}$, let $u(S)$ denote the number of pairs of points in $S$ at distance 1. Define

$$
u_{d}(n)=\max \left\{u(S): S \subset \mathbb{R}^{d},|S|=n\right\} .
$$

Erdôs initiated the study of $u_{2}(n)$ in [4] and of $u_{d}(n)$ in the higher-dimensional case $d \geq 3$ in [5]. The cases $d=2$ and $d=3$ are the most difficult. Erdős [4] obtained

This material is based upon work supported by the South African National Research Foundation.

K.J. Swanepoel ( $₫)$

Fakultät für Mathematik, Technische Universität Chemnitz, 09107 Chemnitz, Germany

e-mail: konrad.swanepoel@gmail.com

K.J. Swanepoel

Department of Mathematical Sciences, University of South Africa, PO Box 392, Pretoria 0003, South Africa 
the superlinear lower bound

$$
u_{2}(n) \geq n^{1+\frac{c}{\log \log n}}
$$

which he conjectured to be tight [9-12]. The best known upper bound is

$$
u_{2}(n) \leq c n^{4 / 3}
$$

due to Spencer, Szemerédi, and Trotter [27]. See Székely [32] for a particularly simple proof.

For $d=3$, the known lower (Erdős [5]) and upper bounds (Clarkson et al. [3]) are the following:

$$
c n^{4 / 3} \log \log n \leq u_{3}(n) \leq c n^{3 / 2} \beta(n),
$$

where $\beta(n)$ is an extremely slowly growing function related to the inverse Ackerman function.

For $d \geq 4$ (the subject of this paper), the situation changes drastically. Lenz, as reported in [5], observed that if we take $p:=\lfloor d / 2\rfloor$ circles in pairwise orthogonal 2dimensional subspaces, each with center at the origin and radius $1 / \sqrt{2}$, then any two points on different circles are at unit distance. Therefore, if $n$ points are chosen by taking $n / p+O(1)$ points on each circle, $\frac{p-1}{2 p} n^{2}-O(1)$ unit distances are obtained. Erdős [5] showed that since $K_{p+1}(3)$, the complete $(p+1)$-partite graph with three vertices in each class, does not occur as a unit-distance graph in $\mathbb{R}^{d}$, the Erdős-Stone theorem [17] gives:

$$
u_{d}(n)=\frac{p-1}{2 p} n^{2}+o\left(n^{2}\right) \quad \text { for all } d \geq 4 .
$$

Using an extremal graph theory result of Erdős [6] and Simonovits [26], Erdős [7] determined the exact value of $u_{d}(n)$ when $d \geq 4$ is even and $n$ is a sufficiently large (depending on $d$ ) multiple of $2 d=4 p$. The $n / p$ points on each circle are then the vertices of $n /(4 p)$ squares. This determines $u_{d}(n)$ asymptotically for all sufficiently large $n$ up to an $O(1)$ term (still for $d$ even). Brass [1] (together with a number theoretical result of van Wamelen [33]) determined $u_{4}(n)$ completely. For $n \geq 5$,

$$
u_{4}(n)= \begin{cases}\left\lfloor n^{2} / 4\right\rfloor+n & \text { if } n \text { is divisible by } 8 \text { or } 10 \\ \left\lfloor n^{2} / 4\right\rfloor+n-1 & \text { otherwise. }\end{cases}
$$

For odd $d \geq 5$, Erdős and Pach [14] showed that

$$
u_{d}(n)=\frac{p-1}{2 p} n^{2}+\Theta\left(n^{4 / 3}\right) .
$$

For the lower bound, they observed that the Lenz construction can be improved when $d$ is odd by replacing one of the circles by a 2 -sphere of radius $1 / \sqrt{2}$ in a 3 -dimensional space orthogonal to the other 2-dimensional subspaces and by placing the points on the sphere such that the unit distance occurs at least $c n^{4 / 3}$ times (a construction of Erdős, Hickerson, and Pach [13]). For the upper bound, they used 
a stability result in extremal graph theory [2, Chap. 5, Remark 4.5(ii)] together with the fact that the maximum number of unit distances among $n$ points on a 2 -sphere is $O\left(n^{4 / 3}\right)[3]$.

\subsection{Diameters}

For a finite subset $S$ of $\mathbb{R}^{d}$, we call a pair of points in $S$ a diameter if their distance equals the diameter of $S$. Let $M(S)$ denote the number of diameters in $S$. Define

$$
M_{d}(n)=\max \left\{M(S): S \subset \mathbb{R}^{d},|S|=n\right\} .
$$

Erdős [4] showed that $M_{2}(n)=n$ for $n \geq 3$. Vázsonyi conjectured, as reported in [4], that $M_{3}(n)=2 n-2$ for $n \geq 4$. This was independently proved by Grünbaum [17], Heppes [18], and Straszewicz [28]. For a new proof, see [30].

As in the case of unit distances, the situation is completely different when $d \geq 4$. Erdős [5] showed that for $d \geq 4, M_{d}(n)=\frac{p-1}{2 p} n^{2}+o\left(n^{2}\right)$, the same asymptotics as $u_{d}(n)$. For other work on this problem by Hadwiger, Lenz, and Yugai, see the survey of Martini and Soltan [22].

\section{New Results}

If $d \geq 4$ is even, let $p=d / 2$, and consider any orthogonal decomposition $\mathbb{R}^{d}=$ $V_{1} \oplus \cdots \oplus V_{p}$ with all $V_{i}$ 2-dimensional. In each $V_{i}$, let $C_{i}$ be the circle with center at the origin $o$ and radius $r_{i}$ such that $r_{i}^{2}+r_{j}^{2}=1$ for all distinct $i$ and $j$. When $d \geq 6$, this implies that each $r_{i}=1 / \sqrt{2}$. For the purposes of Lemma 8 below, we call the $p$ circles $C_{1}, \ldots, C_{p}$ an even-dimensional Lenz system. We define an even-dimensional Lenz configuration to be any translate of a finite subset of $\bigcup_{i=1}^{p} C_{i}$.

If $d \geq 5$ is odd, let $p=\lfloor d / 2\rfloor$, and consider any orthogonal decomposition $\mathbb{R}^{d}=$ $V_{1} \oplus \cdots \oplus V_{p}$ with $V_{1}$ 3-dimensional and all other $V_{i}(i=2, \ldots, p)$ 2-dimensional. Let $\Sigma_{1}$ be the sphere in $V_{1}$ with center $o$ and radius $r_{1}$, and for each $i=2, \ldots, p$, let $C_{i}$ be the circle with center $o$ and radius $r_{i}$ such that $r_{i}^{2}+r_{j}^{2}=1$ for all distinct $i, j$. When $d \geq 7$, necessarily each $r_{i}=1 / \sqrt{2}$. Again, as needed for Lemma 8 , we call the 2-sphere and $p-1$ circles $\Sigma_{1}, C_{2}, \ldots, C_{p}$ an odd-dimensional Lenz system. We define an odd-dimensional Lenz configuration to be any translate of a finite subset of $\Sigma_{1} \cup \bigcup_{i=2}^{p} C_{i}$. (Later we distinguish between weak and strong Lenz configurations as a technical notion inside the proofs. The definition here coincides with a strong Lenz construction in the sequel.)

We call a set $S$ of $n$ points in $\mathbb{R}^{d}$ an extremal set with respect to unit distances [diameters] if $u(S)=u_{d}(n)\left[M(S)=M_{d}(n)\right]$.

Theorem 1 For each $d \geq 4$, there exists $N(d)$ such that all extremal sets of $n \geq N(d)$ points (with respect to unit distances or diameters) are Lenz configurations.

The proof uses a typical technique in extremal graph and hypergraph theory [16, $20,23,26]$ : First prove a stability result for sets that are close to extremal, thus giving 
approximate structural information, and then use extremality to deduce more exact structural information.

For even $d \geq 6$, it is then possible to determine $u_{d}(n)$ exactly. On the other hand, for odd $d \geq 5$, the main obstacle to determine $u_{d}(n)$ is our lack of knowledge of the function $f(m)$ which gives the exact maximum number of unit distances between $m$ points on a 2 -sphere of radius $1 / \sqrt{2}$ (for odd $d \geq 7$ ) and the function $g(m)$ which gives the exact maximum number of unit distances between $m$ points on a 2-sphere of arbitrary radius [13,31] (for $d=5$ ).

Let $t_{p}(n)$ denote the number of edges of the Turán p-partite graph on $n$ vertices. This is the complete $p$-partite graph with $\lfloor n / p\rfloor$ or $\lceil n / p\rceil$ vertices in each class $[2$, Chapter VI]. We do not need the exact value of $t_{p}(n)$, only that

$$
t_{p}(n)=\frac{p-1}{2 p} n^{2}-O(1) .
$$

Corollary 2 Let $d \geq 6$ be even. For all sufficiently large $n$ (depending on $d$ ),

$$
u_{d}(n)= \begin{cases}t_{p}(n)+n-r & \text { if } 0 \leq r \leq p-1, \\ t_{p}(n)+n-p & \text { if } p \leq r \leq 3 p-1, \\ t_{p}(n)+n-2 d+r & \text { if } 3 p \leq r \leq 4 p-1,\end{cases}
$$

where $p=d / 2$, and $r$ is the remainder when dividing $n$ by $4 p=2 d$.

For all $d \geq 4$, it is possible to determine $M_{d}(n)$ exactly if $n$ is large. The most complicated case is $d=5$, where it is necessary to know the maximum number of diameters in a set of $n$ points on a 2 -sphere in $\mathbb{R}^{3}$. For each $n \geq 6$, we construct a set of $n$ points in $\mathbb{R}^{3}$ with $2 n-2$ diameters, all lying on a sphere (see Lemma 7(e) below).

Corollary 3 For all sufficiently large n (depending on d),

$$
\begin{aligned}
& M_{4}(n)= \begin{cases}t_{2}(n)+\lceil n / 2\rceil+1 & \text { if } n \neq 3(\bmod 4), \\
t_{2}(n)+\lceil n / 2\rceil & \text { if } n \equiv 3(\bmod 4) ;\end{cases} \\
& M_{5}(n)=t_{2}(n)+n ; \\
& M_{d}(n)=t_{p}(n)+p \quad \text { for even } d \geq 6, \text { where } p=d / 2 ; \\
& M_{d}(n)=t_{p}(n)+\lceil n / p\rceil+p-1 \quad \text { for odd } d \geq 7, \text { where } p=\lfloor d / 2\rfloor .
\end{aligned}
$$

We use two stability theorems to prove Theorem 1, one for even dimensions and one for odd dimensions.

Theorem 4 For all $\varepsilon>0$ and even $d \geq 4$, there exist $\delta>0$ and $N$ such that any set of $n \geq N$ points in $\mathbb{R}^{d}$ with at least $\left(\frac{p-1}{2 p}-\delta\right) n^{2}$ unit distance pairs can be partitioned into $S_{0}, S_{1}, \ldots, S_{p}$ such that $\left|S_{0}\right|<\varepsilon n$ and for each $i=1, \ldots, p$,

$$
\frac{n}{p}-\varepsilon n<\left|S_{i}\right|<\frac{n}{p}+\varepsilon n
$$


and $S_{i}$ is on a circle $C_{i}$ such that the circles $C_{1}, \ldots, C_{p}$ have the same center and are mutually orthogonal.

Theorem 5 For all $\varepsilon>0$ and odd $d \geq 5$, there exist $\delta>0$ and $N$ such that any set $S$ of $n \geq N$ points in $\mathbb{R}^{d}$ with at least $\left(\frac{p-1}{2 p}-\delta\right) n^{2}$ unit distance pairs can be partitioned into $S_{0}, S_{1}, \ldots, S_{p}$ such that $\left|S_{0}\right|<\varepsilon n$ and for each $i=1, \ldots, p$,

$$
\frac{n}{p}-\varepsilon n<\left|S_{i}\right|<\frac{n}{p}+\varepsilon n,
$$

$S_{1}$ is on a 2-sphere $\Sigma_{1}, S_{i}$ is on a circle $C_{i}, i=2, \ldots, p$, and $\Sigma_{1}, C_{2}, \ldots, C_{p}$ have the same center and are mutually orthogonal.

Corollary 6 Let $d \geq 4$. If a set $S$ of $n$ points in $\mathbb{R}^{d}$ has at least $\left(\frac{p-1}{2 p}-o(1)\right) n^{2}$ unit distance pairs, then $S$ is a Lenz configuration except for $o(n)$ points.

\section{Overview of the Paper}

In Sect. 4 we prove some geometrical lemmas.

In Sect. 5 we determine the maximum number of unit distances and diameters in even-dimensional Lenz configurations, introduce the notions of weak and strong Lenz configuration in odd dimensions, show that the weak Lenz configurations with the largest number of unit distances or diameters are strong Lenz configurations, and determine the maximum number of diameters in strong Lenz configurations. Corollaries 2 and 3 then follow, given that extremal sets are Lenz configurations (weak Lenz configurations when $d \geq 5$ is odd).

In Sect. 6 we use the Erdős-Simonovits stability theorem from extremal graph theory to prove Theorems 4 and 5, from which Corollary 6 is immediate.

Finally, in Sect. 7 we use the stability theorems (Theorems 4 and 5) to show that sets of points that are extremal with respect to unit distances or diameters are (weak) Lenz configurations, thereby finishing the proof of Theorem 1.

\section{Geometric Preliminaries}

We denote the distance between points $a$ and $b$ in $\mathbb{R}^{d}$ by $|a b|$. The unit distance graph of a set $S$ of $n$ points in $\mathbb{R}^{d}$ is defined by joining any two points at distance 1 . Let $u(S)$ denote the number of (unordered) unit distance pairs in $S$. Two points in $S$ at distance 1 are neighbors. For any point $x$ and finite set $S$, let $u(x, S)$ denote the number of points in $S$ that are at distance 1 to $x$. Similarly, for any finite sets $A$ and $B$, let $u(A, B)$ denote the number of (ordered) unit distance pairs $(a, b)$ with $a \in A$ and $b \in B$.

Whenever we work with diameters, we assume that the diameter of $S$ is 1 and then we use the notation $u(S), u(x, S)$, and $u(A, B)$ as before. In this case we call the unit distance graph of $S$ the diameter graph of $S$. 
We continually use the following two basic lemmas in the sequel. The first deals with unit distances and diameters on circles and 2-spheres, and the second one with unit distances in dimensions higher than 3.

Lemma 7 Let $S$ be a set of $n$ points in $\mathbb{R}^{3}$.

(a) If $S$ lies on a circle of radius $1 / \sqrt{2}$, then

$$
u(S) \leq \begin{cases}n & \text { if } n \text { is divisible by } 4 \\ n-1 & \text { otherwise }\end{cases}
$$

Equality is possible for all $n$ by letting $S$ be the union of the vertices of $\lfloor n / 4\rfloor$ inscribed squares and $n-4\lfloor n / 4\rfloor$ vertices of an additional square.

(b) If $S$ has diameter 1 and lies on a circle, then

$$
u(S) \leq \begin{cases}n & \text { if } n \text { is odd } \\ n-1 & \text { if } n \text { is even }\end{cases}
$$

Equality is possible for all $n \geq 2$, for a circle of suitable radius depending on $n$.

(c) If $S$ has diameter 1 and lies on a circle of radius $>1 / \sqrt{3}$, then $u(S)=1$.

(d) If $S$ lies on a 2-sphere, then $u(S)=O\left(n^{4 / 3}\right)$. When the radius equals $1 / \sqrt{2}$, there exists a set $S$ with $u(S)=\Omega\left(n^{4 / 3}\right)$.

(e) If $S$ has diameter 1 and lies on a 2-sphere, then $u(S) \leq 2 n-2$. Equality is possible for each $n \geq 4, n \neq 5$, for a 2 -sphere of suitable radius depending on $n$.

(f) If $S$ has diameter 1 and lies on a 2-sphere of radius $\geq 1 / \sqrt{2}$, then $u(S) \leq n$. Equality is possible for all $n \geq 3$ and all radii $\geq 1 / \sqrt{2}$.

Proof Statements (a), (b), and (c) are straightforward, except perhaps that $u(S) \leq$ $n-1$ for an even number of concyclic points of diameter 1 . This follows essentially from the easily seen observation that if the diameter graph of points of some concyclic points contains a cycle, then it consists only of this cycle, together with the wellknown fact that all cycles in diameter graphs in the plane are odd [19, 29].

The upper bound in (d) is due to Clarkson et al. [3]. The simplest known proof of it is by adapting Székely's proof [32] for the planar case. The lower bound in (d) is due to Erdôs, Hickerson, and Pach [13].

Statement (f) can be found in Kupitz, Martini, and Wegner [21]. It follows as in the planar case [25, Theorem 13.13] from the observation that any two diameters, when drawn as short great circular arcs on the 2-sphere, must intersect. Examples of $n$ points with $n$ diameters are easily found for all radii larger than $1 / \sqrt{2}$; they have essentially the same structure as in the plane; see [21] for details.

The upper bound of $2 n-2$ in (e) is the Grünbaum-Heppes-Straczewicz upper bound for diameters in $\mathbb{R}^{3}$ [25, Theorem 13.14]. (For a new proof, see [30].) The following is a short proof for points on a 2 -sphere. For a point $x$ on the sphere, denote its opposite point by $x^{\prime}$. Colour the $n$ given points blue and their opposite points red. For any diameter $x y$, join the blue point $x$ and the red point $y^{\prime}$ by a short arc of the great circle passing through them and do the same with $x^{\prime}$ and $y$. This defines a 
Fig. 1 Circle $C$ with points $x_{1}$ to $x_{n-3}$

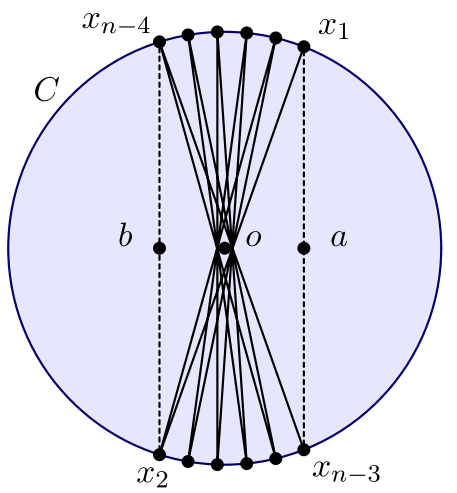

bipartite geometric graph on the sphere, with all the arcs of the same length $r$, say. It is easily seen that this graph is planar: if the arcs $a b^{\prime}$ and $c d^{\prime}$ intersect, then by the triangle inequality the arc $a d^{\prime}$ or the $\operatorname{arc} b^{\prime} c$ will be shorter than $r$. Then either $|a d|$ or $|b c|$ will be larger than the diameter, a contradiction. This graph has $2 n$ vertices. By Euler's formula, a bipartite planar graph on $2 n$ vertices has at most $4 n-4$ edges. Since this is twice the number of diameters, the upper bound follows.

The only statement that remains to be proved is that $2 n-2$ diameters can be attained on a 2 -sphere for each $n \geq 4, n \neq 5$. For even $n \geq 4$, the following construction is described in Neaderhouser and Purdy [24]. Consider the vertex set of a regular $(n-1)$-gon of diameter 1 and choose another point on the axis of symmetry of the polygon at distance 1 to the $n-1$ vertices. This gives $n$ points with $2 n-2$ diameters.

For odd $n \geq 7$, a more involved construction is needed. Place $n-3$ points $x_{1}, \ldots, x_{n-3}$ on the circle $C$ of radius $r$ and center $o$ in the $x y$-plane such that the diameter 1 occurs between consecutive $x_{i}$ 's (Fig. 1).

Note that $r$ and $n$ determine everything up to isometry. We fix $r$ later in the proof. Let $x_{n-2}$ be the point on the positive $z$-axis at distance 1 to each point of $C$. Then $x_{n-2}$ and $C$ are on a unique sphere $\Sigma$ with center $o^{\prime}$ and radius $s$, say. Note that $o^{\prime}$ is on the positive $z$-axis.

We now want to find points $x_{n-1}$ and $x_{n}$ on $\Sigma$ such that

$$
\left|x_{1} x_{n-1}\right|=\left|x_{n-3} x_{n-1}\right|=\left|x_{2} x_{n}\right|=\left|x_{n-4} x_{n}\right|=\left|x_{n-1} x_{n}\right|=1
$$

and

$$
\left|x_{n-2} x_{n-1}\right| \leq 1, \quad\left|x_{n-2} x_{n}\right| \leq 1
$$

as in Fig. 2.

This will give $2 n-2$ diameters in the set $S:=\left\{x_{1}, \ldots, x_{n}\right\}$. For any value of $r$, there will clearly be unique points $x_{n-1}, x_{n} \in \Sigma \backslash\left\{x_{n-2}\right\}$ that satisfy

$$
\left|x_{n-3} x_{n-1}\right|=\left|x_{1} x_{n-1}\right|=\left|x_{2} x_{n}\right|=\left|x_{n-4} x_{n}\right|=1 \text {. }
$$

It remains to find an appropriate value of $r$ so that

$$
\left|x_{n-1} x_{n}\right|=1, \quad\left|x_{n-2} x_{n-1}\right| \leq 1, \quad\left|x_{n-2} x_{n}\right| \leq 1 \text {. }
$$


Fig. 215 points with 28 diameters on a sphere

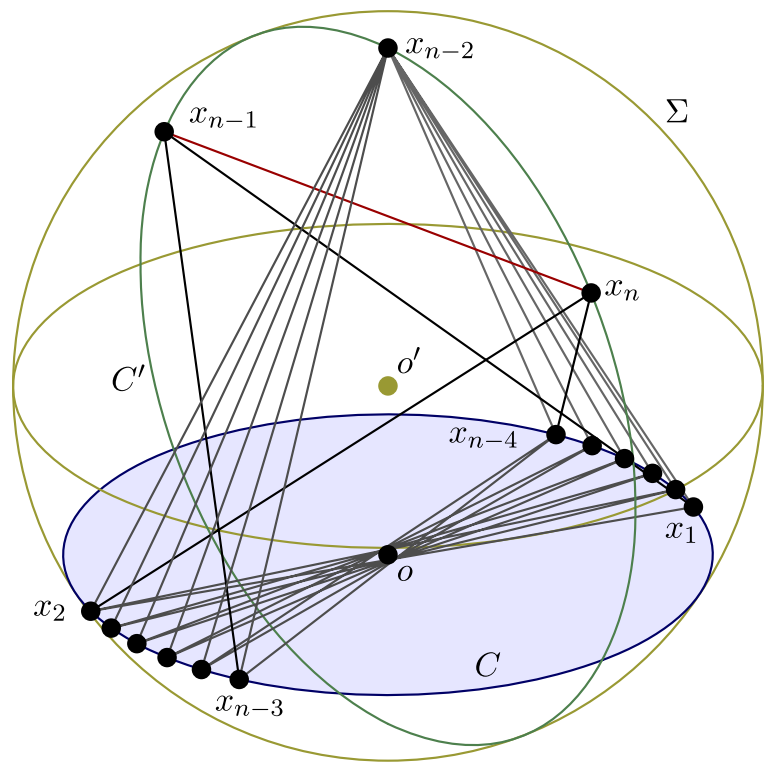

Fig. 3 Circle $C^{\prime}$

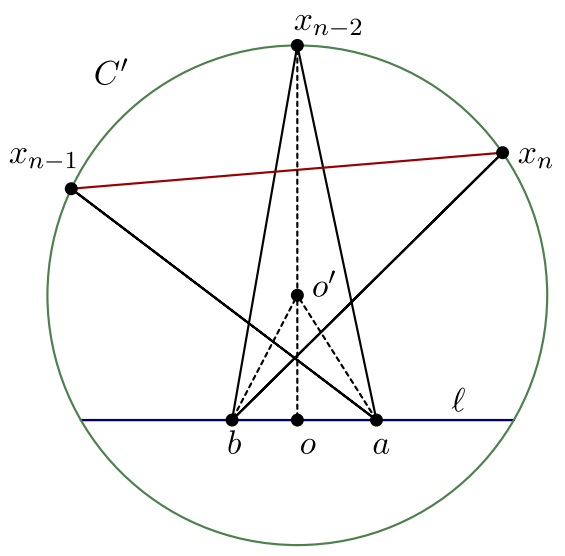

We reduce this to a two-dimensional problem (Fig. 3). Let $a$ and $b$ be the midpoints of $x_{1} x_{n-3}$ and $x_{2} x_{n-4}$, respectively. Consider the intersection of $\Sigma$ with the plane $o a b x_{n-2}$. This is a circle $C^{\prime}$ with center $o^{\prime}$ and radius $s$. By symmetry, $x_{n-1}$ and $x_{n}$ lie on $C^{\prime}$, and $\left|a x_{n-2}\right|=\left|a x_{n-1}\right|$ and $\left|b x_{n-2}\right|=\left|b x_{n}\right|$. Therefore, $a o^{\prime}$ bisects $\varangle x_{n-2} a x_{n-1}$, and $b o^{\prime}$ bisects $\varangle x_{n-2} b x_{n}$. Clearly, $|o a|>|o b|$, and both $|o a|$ and $|o b|$ are strictly monotone functions of $r$.

We now consider $r$ to be a variable ranging in the interval $\left(1 / 2, r_{0}\right)$, where

$$
r_{0}:=\left(2 \cos \frac{\pi}{2(n-4)}\right)^{-1}
$$


On the one hand, $r>\frac{1}{2}$, and in the limit, as $r \rightarrow \frac{1}{2}$, the diameters $x_{i} x_{i+1}$ all coincide, and $\lim _{r \rightarrow 1 / 2}|o a|=\lim _{r \rightarrow 1 / 2}|o b|=0$. It follows that

$$
\lim _{r \rightarrow 1 / 2}\left|x_{n-2} x_{n-1}\right|=\lim _{r \rightarrow 1 / 2}\left|x_{n-2} x_{n}\right|=0,
$$

hence

$$
\lim _{r \rightarrow 1 / 2}\left|x_{n-1} x_{n}\right|=0 .
$$

On the other hand, $r<r_{0}$, where in the limit, as $r \rightarrow r_{0}, x_{1}$ and $x_{n-3}$ coincide, and the points form the vertex set of a regular $(n-4)$-gon. Thus

$$
\begin{gathered}
\lim _{r \rightarrow r_{0}}|o a|=r_{0}, \\
\lim _{r \rightarrow r_{0}}|o b| \rightarrow 2 r_{0} \sin \frac{\pi}{n-4},
\end{gathered}
$$

and

$$
\lim _{r \rightarrow r_{0}}\left|x_{n-2} a\right|=1 \text {. }
$$

Since $2 r_{0}>1, \lim _{r \rightarrow r_{0}} x_{n-1}$ is a point below the chord $\ell$ of $C^{\prime}$ through $a$ and $b$. (Note that $\ell$ is a diameter of $C$.) Also,

$$
\lim _{r \rightarrow r_{0}}\left|x_{2} a\right|=\lim _{r \rightarrow r_{0}}\left|x_{n-4} a\right|=1
$$

hence $\lim _{r \rightarrow r_{0}} x_{n}=a$. Since $x_{n-1}$ is lower than $x_{n}$ (because $|o a|>|o b|$ ), $x_{n-1}$ reaches $\ell$ before $x_{n}$. Since $\left|x_{n} b\right|=\left|x_{n-2} b\right|$, the chord $x_{n} b$ is below $o^{\prime}$. Since at this stage (with $x_{n-1} \in \ell$ ) the chord $b x_{n-1}$ is below $o^{\prime}$, the chord $x_{n-1} x_{n}$ is also below $o^{\prime}$. Thus, before $x_{n-1}$ reaches $\ell$, there is a stage where $x_{n-1} x_{n}$ passes through $o^{\prime}$ with both $x_{n-1}$ and $x_{n}$ still above $\ell$, and therefore their distances to $x_{n-2}$ are at most 1. From $s>r>1$ it follows that $\left|x_{n-1} x_{n}\right|>1$. By (1), at some earlier stage, $\left|x_{n-1} x_{n}\right|<1$. Therefore, at some inbetween stage, $\left|x_{n-1} x_{n}\right|=1$. This finishes the construction for odd $n \geq 7$.

We remark that the exception $n \neq 5$ in Lemma 7(f) is genuine. Suppose that there exist 5 points on a 2 -sphere with 8 diameters. Then one of the points must be incident to 4 diameters. The other 4 points are then concyclic, and among them there can be at most 3 diameters (Lemma 7(b)), a contradiction.

Lemma 8 Let $d \geq 4$, and set $p:=\lfloor d / 2\rfloor$.

(a) Suppose that $A_{1}, \ldots, A_{p} \subseteq \mathbb{R}^{d}$ are given such that $\left|A_{i}\right| \geq 3$ for each $i$, and $|a b|=1$ for all $a \in A_{i}$ and $b \in A_{j}$ with distinct $i$ and $j$. Then the $A_{i}$ are contained in some translate of a Lenz system, i.e., there exists a Lenz system and $v \in \mathbb{R}^{d}$ such that after some relabeling of the indices, $A_{i}-v \subseteq C_{i}$ for $i \geq 2$, and $A_{1}-v \subseteq C_{1}$ or $A_{1}-v \subseteq \Sigma_{1}$ depending on whether $d$ is even or odd. 
(b) There does not exist $p+1$ sets $A_{1}, \ldots, A_{p+1} \subseteq \mathbb{R}^{d}$ each containing at least 3 points such that any two points in different sets are at distance 1 .

(c) Suppose that the point $x \in \mathbb{R}^{d}$ is at distance 1 to at least three points of each of the circles $C_{i}, i \geq 2$, in some Lenz system. Then $x \in C_{1}$ if $d$ is even, or $x \in \Sigma_{1}$ if $d$ is odd.

(d) Let $d \geq 7$ be odd. Let $C$ be a circle on $\Sigma_{1}$ in some Lenz system with $v \in V_{1}$ perpendicular to $C$. Suppose that the point $x \in \mathbb{R}^{d}$ is at distance 1 to at least three points of each of the circles $C$ and $C_{i}, i \geq 3$. Then $x$ is on the sphere with center $o$ and radius $1 / \sqrt{2}$ in the 3-dimensional space spanned by $V_{2}$ and $v$.

Proof In this proof we denote the affine hull of a set $A$ by aff $(A)$. Statements (c) and (d) are easy to prove. Note that if a point is at the same distance to 3 points on a circle, then its orthogonal projection on the affine hull of the circle is the center of the circle.

Statement (b) was originally observed by Erdôs [5]. In fact, both (b) and (a) follow from the easily seen observation that if $A, B \subseteq \mathbb{R}^{d}$ are such that $|A|,|B| \geq 3$ and $|a b|=1$ for all $a \in A, b \in B$, then $A$ and $B$ lie on unique spheres in $\operatorname{aff}(A)$ and $\operatorname{aff}(B)$, respectively, and these affine spaces are orthogonal. However, $\operatorname{aff}(A)$ and $\operatorname{aff}(B)$ need not intersect, so the only difficulty is perhaps to prove the existence of $v$ in (a).

Consider, for example, the case of odd $d$ in (a). The pairwise orthogonality of all aff $\left(A_{i}\right)$ implies that $p-1$ of them have dimension 2 , and one has dimension 3 . Without loss of generality, let $\operatorname{dim} \operatorname{aff}\left(A_{1}\right)=3$.

We show that any two $\operatorname{aff}\left(A_{i}\right)$ intersect. Suppose that $\operatorname{aff}\left(A_{1}\right) \cap \operatorname{aff}\left(A_{2}\right)=\emptyset$. Then $\operatorname{dim} \operatorname{aff}\left(A_{1} \cup A_{2}\right)=6$, and since $\operatorname{aff}\left(A_{1} \cup A_{2}\right)$ and $\operatorname{aff}\left(A_{i}\right), i \geq 3$, are pairwise orthogonal, we obtain a contradiction with the dimension. It follows similarly that any other pair of affine hulls has nonempty intersection.

Thus aff $\left(A_{1}\right) \cap \operatorname{aff}\left(A_{2}\right)=\{v\}$, say. It remains to show that all other $\operatorname{aff}\left(A_{i}\right)$ also contain $v$. Suppose, for example, that $v \notin \operatorname{aff}\left(A_{3}\right)$. Then $\operatorname{aff}\left(A_{1}\right) \cap \operatorname{aff}\left(A_{3}\right)=\left\{v_{1}\right\}$ and $\operatorname{aff}\left(A_{2}\right) \cap \operatorname{aff}\left(A_{3}\right)=\left\{v_{2}\right\}$ with $v_{1}, v_{2} \neq v$. Furthermore, $v_{1} \neq v_{2}$, otherwise $\operatorname{aff}\left(A_{1}\right) \cap$ $\operatorname{aff}\left(A_{2}\right)$ would contain more than one point. Thus $\operatorname{aff}\left(A_{1} \cup A_{2}\right) \cap \operatorname{aff}\left(A_{3}\right)$ contains a line. This contradicts the orthogonality of $\operatorname{aff}\left(A_{1} \cup A_{2}\right)$ and $\operatorname{aff}\left(A_{3}\right)$.

The case where $d$ is even is similar.

\section{Optimized Lenz Configurations}

\subsection{Even Dimensions $d \geq 6$}

We have already defined a Lenz configuration in the introduction. For any Lenz configuration $S$ on $n$ points lying on $p=d / 2$ mutually orthogonal circles $C_{i}$ with center $o$ and radius $1 / \sqrt{2}$, we define $S_{i}:=S \cap C_{i}$ and $n_{i}:=\left|S_{i}\right|$.

\subsubsection{Unit Distances}

When $d \geq 6$ is even, define

$$
u_{d}^{L}(n)=\max \left\{u(S): S \text { is a Lenz configuration of } n \text { points in } \mathbb{R}^{d}\right\} .
$$


We call any Lenz configuration $S$ of $n$ points in $\mathbb{R}^{d}$ for which $u(S)=u_{d}^{L}(n)$ an optimized Lenz configuration (for unit distances).

Proposition 9 Let $d \geq 6$ be even, $n \geq 1, p=d / 2$, and $n \equiv r(\bmod 2 d), 0 \leq r \leq$ $2 d-1$. Then

$$
u_{d}^{L}(n)= \begin{cases}t_{p}(n)+n-r & \text { if } 0 \leq r \leq p-1, \\ t_{p}(n)+n-p & \text { if } p \leq r \leq 3 p-1 \\ t_{p}(n)+n-2 d+r & \text { if } 3 p \leq r \leq 4 p-1\end{cases}
$$

Proof Consider an optimized Lenz configuration $S$ on $p$ pairwise orthogonal circles $C_{1}, \ldots, C_{p}$. We may rearrange the points on each circle without changing the number of unit distances between circles. By Lemma 7(a) and maximality, each $u\left(S_{i}\right)=n_{i}$ if $n_{i} \equiv 0(\bmod 4)$ and $u\left(S_{i}\right)=n_{i}-1$ otherwise. The problem is now that of maximizing the function

$$
u\left(n_{1}, \ldots, n_{p}\right):=\left(\sum_{1 \leq i<j \leq p} n_{i} n_{j}\right)+n-p+k\left(n_{1}, \ldots, n_{p}\right)
$$

over all nonnegative $n_{1}, \ldots, n_{p}$ that sum to $n$, where $k\left(n_{1}, \ldots, n_{p}\right)$ equals the number of $n_{i}$ divisible by 4 . This easy but tedious exercise finishes the proof.

\subsubsection{Diameters}

Still for even $d \geq 6$, define

$$
M_{d}^{L}(n)=\max \left\{M(S): S \text { is a Lenz configuration of } n \text { points in } \mathbb{R}^{d}\right\} .
$$

We call any diameter 1 Lenz configuration $S$ of $n$ points in $\mathbb{R}^{d}$ for which $M(S)=$ $u(S)=M_{d}^{L}(n)$ an optimized Lenz configuration (for diameters).

Proposition 10 Let $d \geq 6$ be even, $n \geq d$, and $p=d / 2$. Then

$$
M_{d}^{L}=t_{p}(n)+p
$$

Proof Consider an optimized Lenz configuration $S$ of diameter 1 on $p$ pairwise orthogonal circles $C_{1}, \ldots, C_{p}$. By Lemma $7(\mathrm{c})$, each $u\left(S_{i}\right) \leq 1$. Therefore, $u(S) \leq$ $t_{p}(n)+p$. Equality is clearly possible if $n \geq d$ by dividing the $n$ points as equally as possible between the $p$ circles and ensuring that a diameter occurs within each $S_{i}$.

\subsection{The Dimension $d=4$}

For any Lenz configuration $S$ on $n$ points lying on orthogonal circles $C_{1}$ and $C_{2}$ with common center $o$ and radii $r_{1}$ and $r_{2}$ such that $r_{1}^{2}+r_{2}^{2}=1$, define $S_{i}:=S \cap C_{i}$ and $n_{i}:=\left|S_{i}\right|$. 


\subsubsection{Unit Distances}

This section is included for the sake of completeness. As for even $d \geq 6$, define

$$
u_{4}^{L}(n)=\max \left\{u(S): S \text { is a Lenz configuration of } n \text { points in } \mathbb{R}^{4}\right\} .
$$

As shown by Brass [1] and van Wamelen [33]:

Proposition 11 Let $n \geq 5$. Then

$$
u_{4}^{L}(n)= \begin{cases}t_{2}(n)+n & \text { if } n \text { is divisible by } 8 \text { or } 10 \\ t_{2}(n)+n-1 & \text { otherwise }\end{cases}
$$

\subsubsection{Diameters}

As for even $d \geq 6$, define

$$
M_{4}^{L}(n)=\max \left\{u(S): S \text { is a Lenz configuration of } n \text { points in } \mathbb{R}^{4}\right\} .
$$

We call any diameter 1 Lenz configuration $S$ of $n$ points in $\mathbb{R}^{4}$ for which $M(S)=$ $u(S)=M_{4}^{L}(n)$ an optimized Lenz configuration (for diameters).

Proposition 12 Let $n \geq 6$. Then

$$
M_{4}^{L}(n)= \begin{cases}t_{2}(n)+\lceil n / 2\rceil+1 & \text { if } n \neq 3(\bmod 4), \\ t_{2}(n)+\lceil n / 2\rceil & \text { if } n \equiv 3(\bmod 4) .\end{cases}
$$

Proof Consider an optimized Lenz configuration $S$ of diameter 1 on pairwise orthogonal circles $C_{1}$ and $C_{2}$. Without loss of generality, $r_{1} \leq r_{2}$. We now apply Lemma 7(b), (c). If $u\left(S_{2}\right)>1$, then $r_{2} \leq 1 / \sqrt{3}$, hence $r_{1} \geq \sqrt{2 / 3}>r_{2}$, a contradiction. Therefore, $u\left(S_{2}\right) \leq 1$. Also, $u\left(S_{1}\right) \leq n_{1}$, and if $n_{1}$ is even, $u\left(S_{1}\right) \leq n_{1}-1$. It follows that

$$
u(S) \leq \begin{cases}n_{1} n_{2}+n_{1}+1 & \text { if } n_{1} \text { is odd } \\ n_{1} n_{2}+n_{1} & \text { if } n_{1} \text { is even. }\end{cases}
$$

By considering the four cases of $n$ modulo 4 , it is easily checked that the maximum over all nonnegative $n_{i}$ with $n_{1}+n_{2}=n$ is as in the statement of the theorem. For $n \geq 6$, it is also easy to see that there are configurations that attain this maximum.

\subsection{Odd Dimensions $d \geq 7$}

We introduce the notion of a weak Lenz configuration. Let $d \geq 7$ be odd, $p=(d-$ 1) $/ 2$, and consider any orthogonal decomposition $\mathbb{R}^{d}=V_{0} \oplus V_{1} \oplus \cdots \oplus V_{p}$ with $\operatorname{dim} V_{0}=1$ and $\operatorname{dim} V_{i}=2(i=1, \ldots, p)$. For each $i=1, \ldots, p$, let $\Sigma_{i}$ be the sphere in $V_{0} \oplus V_{i}$ with center $o$ and radius $1 / \sqrt{2}$, and let $C_{i}$ be the circle $V_{i} \cap \Sigma_{i}$. Let $p^{+}$ and $p^{-}$be the two points in $V_{0}$ at distance $1 / \sqrt{2}$ from $o$. Thus $p^{+}$and $p^{-}$are the north and south poles of each $\Sigma_{i}$ when $C_{i}$ is considered to be its equator. 
A strong Lenz configuration of $n$ points in $\mathbb{R}^{d}$ is a translate of a finite subset of $C_{1} \cup \cdots \cup C_{p-1} \cup \Sigma_{p}$ for some orthogonal decomposition. (This is merely the odd-dimensional "Lenz configuration" of Sect. 2.) A weak Lenz configuration of $n$ points in $\mathbb{R}^{d}$ is a translate of a finite subset of a $\Sigma_{1} \cup \cdots \cup \Sigma_{p}$ for some orthogonal decomposition. Strong Lenz configurations are clearly also weak. If $S$ is a weak Lenz configuration, we assume without loss of generality that it is a subset of $\Sigma_{1} \cup \cdots \cup \Sigma_{p}$ and we define $S_{i}:=S \cap \Sigma_{i} \backslash\left\{p^{+}, p^{-}\right\}(i=1, \ldots, p), S_{0}:=S \cap\left\{p^{+}, p^{-}\right\}, n_{i}:=\left|S_{i}\right|$ $(i=0, \ldots, p), n:=|S|$.

\subsubsection{Unit Distances}

For odd $d \geq 7$, define

$$
u_{d}^{L}(n)=\max \left\{u(S): S \text { is a weak Lenz configuration of } n \text { points in } \mathbb{R}^{d}\right\} .
$$

We call any weak Lenz configuration $S$ of $n$ points in $\mathbb{R}^{d}$ for which $u(S)=u_{d}^{L}(n)$ an optimized Lenz configuration (for unit distances). Unlike the even-dimensional case, we cannot give an expression for $u_{d}^{L}(n)$ more accurate than the estimate $u_{d}^{L}(n)=t_{p}(n)+\Theta\left(n^{4 / 3}\right)$ of Erdôs and Pach [14]. However, we next show that an optimized Lenz configuration must be strong for $n$ sufficiently large depending on $d$. This implies that $u_{d}^{L}(n)$ can be determined if the function $f(n)$, which gives the maximum number of unit distances for $n$ points on a 2 -sphere of radius $1 / \sqrt{2}$, is known.

Lemma 13 For any distinct $i$ and $j$, no point of $\Sigma_{i} \backslash C_{i}$ can be at unit distance to any point of $\Sigma_{j} \backslash C_{j}$.

Proof Choose $x \in \Sigma_{i}$ and $y \in \Sigma_{j}$. Then $x=\lambda_{i} p^{+}+v_{i}$ and $y=\lambda_{j} p^{+}+v_{j}$ for unique $\lambda_{i}, \lambda_{j} \in \mathbb{R}$ and $v_{i} \in V_{i}, v_{j} \in V_{j}$. Then $\langle x, y\rangle=\lambda_{i} \lambda_{j} / 2$, hence $|x y|^{2}=\|x\|^{2}-$ $2\langle x, y\rangle+\|y\|^{2}=1-\lambda_{i} \lambda_{j}$. If $x \notin C_{i}$ and $y \notin C_{j}$, then $\lambda_{i}, \lambda_{j} \neq 0$ and $|x y| \neq 1$.

Proposition 14 For each odd $d \geq 7$, there exists $N(d)$ such that all optimized Lenz configurations for unit distances on $n \geq N(d)$ points in $\mathbb{R}^{d}$ are strong Lenz configurations.

Proof Let $S$ be an optimized Lenz configuration on $n$ points. Suppose that $S$ is not a strong Lenz configuration. Thus, without loss of generality, $S_{i} \backslash C_{i} \neq \emptyset$ for $i=1,2$. We aim for a contradiction.

Since $u\left(S_{1} \backslash C_{1}\right)=O\left(\left|S_{1} \backslash C_{1}\right|^{4 / 3}\right)($ Lemma $7(\mathrm{~d}))$ and $S_{1} \backslash C_{1} \neq \emptyset$, there exists $x \in S_{1} \backslash C_{1}$ with $u\left(x, S_{1} \backslash C_{1}\right)=O\left(\left|S_{1} \backslash C_{1}\right|^{1 / 3}\right)=O\left(n^{1 / 3}\right)$. Also, since $x \neq p^{ \pm}$, $u\left(x, C_{1}\right) \leq 2$. Therefore, $u\left(x, S_{1}\right)=O\left(n^{1 / 3}\right)$. Note that for each $i=2, \ldots, p, x$ is at distance 1 to all points in $S_{i} \cap C_{i}$ but, by Lemma 13, to none of $S_{i} \backslash C_{i}$. If we replace $x$ by a new point on $C_{1}$, we lose at most $u\left(x, S_{1}\right)$ unit distances and gain $\sum_{i=2}^{p}\left|S_{i} \backslash C_{i}\right|$. Since $u(S)$ is the maximum over all weak Lenz configurations,

$$
\sum_{i=2}^{p}\left|S_{i} \backslash C_{i}\right| \leq u\left(x, S_{1}\right)=O\left(n^{1 / 3}\right) .
$$


By instead considering a point $x \in S_{2} \backslash C_{2}$ we similarly obtain that

$$
\sum_{\substack{i=1 \\ i \neq 2}}^{p}\left|S_{i} \backslash C_{i}\right|=O\left(n^{1 / 3}\right)
$$

Therefore, $\left|S_{i} \backslash C_{i}\right|=O\left(n^{1 / 3}\right)$ for each $i=1, \ldots, p$.

We can now bound $u(S)$ from above. Note that each point of $S_{0}$ is at unit distance to each point of $C_{i}$, but to none of $\Sigma_{i} \backslash C_{i}$, each point of $\Sigma_{i} \backslash\left\{p^{+}, p^{-}\right\}$is at unit distance to at most two points of $C_{i}, u\left(S_{i} \cap C_{i}\right) \leq\left|S_{i} \cap C_{i}\right|$ (Lemma 7(a)), and $u\left(S_{i} \backslash\right.$ $\left.C_{i}\right)=O\left(\left|S_{i} \backslash C_{i}\right|^{4 / 3}\right)($ Lemma $7(\mathrm{~d}))$. This gives:

$$
\begin{aligned}
u\left(S_{i}\right) & \leq u\left(S_{0} \cup S_{i}\right) \\
& =u\left(S_{0}, S_{i}\right)+u\left(S_{i} \cap C_{i}\right)+u\left(S_{i} \cap C_{i}, S_{i} \backslash C_{i}\right)+u\left(S_{i} \backslash C_{i}\right) \\
& \leq 2\left|S_{i} \cap C_{i}\right|+\left|S_{i} \cap C_{i}\right|+2\left|S_{i} \backslash C_{i}\right|+O\left(\left|S_{i} \backslash C_{i}\right|^{4 / 3}\right) \\
& =O(n)+O\left(\left(n^{1 / 3}\right)^{4 / 3}\right)=O(n) .
\end{aligned}
$$

Therefore, (grouping $S_{0}$ and $S_{1}$ together)

$$
\begin{aligned}
u(S) & \leq t_{p}(n)+u\left(S_{0} \cup S_{1}\right)+\sum_{i=2}^{p} u\left(S_{i}\right) \\
& =t_{p}(n)+O(n),
\end{aligned}
$$

which contradicts $u(S)=u_{d}^{L}(n)=t_{p}(n)+\Theta\left(n^{4 / 3}\right)$ for large $n$.

\subsubsection{Diameters}

For odd $d \geq 7$, define

$$
M_{d}^{L}(n)=\max \left\{M(S): S \text { is a weak Lenz configuration of } n \text { points in } \mathbb{R}^{d}\right\} .
$$

We call any diameter 1 weak Lenz configuration $S$ of $n$ points in $\mathbb{R}^{d}$ for which $M(S)=u(S)=M_{d}^{L}(n)$ an optimized Lenz configuration (for diameters). We show that an optimized Lenz configuration must be strong for large $n$, and furthermore determine the exact value of $M_{d}^{L}(n)$.

Proposition 15 For each odd $d \geq 7$, there exists $N(d)$ such that all optimized Lenz configurations for diameters on $n \geq N(d)$ points in $\mathbb{R}^{d}$ are strong Lenz configurations. Furthermore,

$$
M_{d}^{L}(n)=t_{p}(n)+\left\lceil\frac{n}{p}\right\rceil+p-1=t_{p}(n-1)+n-1+p .
$$

Proof Choose a set $S$ of $n$ points equally distributed between the orthogonal circles $C_{1}, \ldots, C_{p-1}$ and 2-sphere $\Sigma_{p}$ such that the diameter of each $S \cap C_{i}$ is 1 and also 
$S \cap \Sigma_{p} \backslash C_{p}=\left\{p^{+}\right\}$and $\left|S \cap C_{p}\right|=\lceil n / p\rceil-1$. Then clearly $u(S)=t_{p}(n)+\lceil n / p\rceil+$ $p-1$. Therefore, $M_{d}^{L}(n) \geq t_{p}(n)+\lceil n / p\rceil+p-1$. We need this lower bound in a moment.

Now let $S$ be any optimized Lenz configuration on $n$ points. Let $k_{i}:=\left|S_{i} \backslash C_{i}\right|$ $(i=1, \ldots, p)$. We have to show that $S$ is a strong Lenz configuration, i.e., that $k_{i}=0$ for all $i=1, \ldots, p$ except at most one.

First consider the case where $S_{0} \neq \emptyset$; without loss of generality, $S_{0}=\left\{p^{+}\right\}$. Then

$$
\begin{aligned}
u(S) & =u\left(S \backslash\left\{p^{+}\right\}\right)+\sum_{i=1}^{p} u\left(p^{+}, S_{i}\right) \\
& =\sum_{1 \leq i<j \leq p} u\left(S_{i}, S_{j}\right)+\sum_{i=1}^{p} u\left(S_{i}\right)+\sum_{i=1}^{p} u\left(p^{+}, S_{i}\right) \\
& =\sum_{1 \leq i<j \leq p} u\left(S_{i}, S_{j}\right)+\sum_{i=1}^{p} u\left(S_{i} \cup\left\{p^{+}\right\}\right) \\
& =\sum_{1 \leq i<j \leq p}\left|S_{i}\right|\left|S_{j}\right|-\sum_{1 \leq i<j \leq p} k_{i} k_{j}+\sum_{i=1}^{p} u\left(S_{i} \cup\left\{p^{+}\right\}\right) \quad(\mathrm{L} \\
& \leq t_{p}(n-1)-\sum_{1 \leq i<j \leq p} k_{i} k_{j}+\sum_{i=1}^{p}\left(n_{i}+1\right) \quad(\text { Lemma } 7(\mathrm{f})) \\
& =t_{p}(n-1)-\sum_{1 \leq i<j \leq p} k_{i} k_{j}+n-1+p \\
& =t_{p}(n)+\lceil n / p\rceil+p-1-\sum_{1 \leq i<j \leq p} k_{i} k_{j} .
\end{aligned}
$$

However, $u(S)=M_{d}^{L}(n) \geq t_{p}(n)+\lceil n / p\rceil+p-1$, hence $\sum_{1 \leq i<j \leq p} k_{i} k_{j}=0$, which implies that $k_{i}=0$ for all $i$ except one. This finishes the case $S_{0} \neq \varnothing$.

Next consider the case where $S_{0}=\emptyset$. Without loss of generality, $S_{1} \backslash C_{1} \neq \emptyset$, otherwise, by Proposition $10, u(S) \leq t_{p}(n)+p$, a contradiction for large $n$. By Lemma 7(f), $u\left(S_{1}\right) \leq n_{1}$. If we remove the points in $S_{1}$, place $p^{+}$into $S_{0}$ and choose $n_{1}-1$ new points of diameter 1 on $C_{1}$ to form another set $S^{\prime}$ of diameter 1 , we then lose at most $n_{1}$ diameters and gain $n_{1}+k_{1} \sum_{i=2}^{p} k_{i}$. By maximality, $\sum_{i=2}^{p} k_{i}=0$, i.e., the original $S$ was already a strong Lenz configuration and $u(S)=u\left(S^{\prime}\right)$. Since $p^{+} \in S^{\prime}$, this case is reduced to the previous one. The theorem is proved.

\subsection{The Dimension $d=5$}

Consider an orthogonal decomposition $\mathbb{R}^{5}=V_{0} \oplus V_{1} \oplus V_{2}$ such that $\operatorname{dim} V_{0}=1$ and $\operatorname{dim} V_{1}=\operatorname{dim} V_{2}=2$. Choose $r_{1} \in(0,1)$. Let $\Sigma_{1}$ be the 2-sphere in $V_{0} \oplus V_{1}$ with center $o$ and radius $r_{1}$. Let $C_{2}$ be the circle in $V_{2}$ with center $o$ and radius $r_{2}:=\sqrt{1-r_{1}^{2}}$. Then any point of $\Sigma_{1}$ and any point of $C_{2}$ are at unit distance. We call 


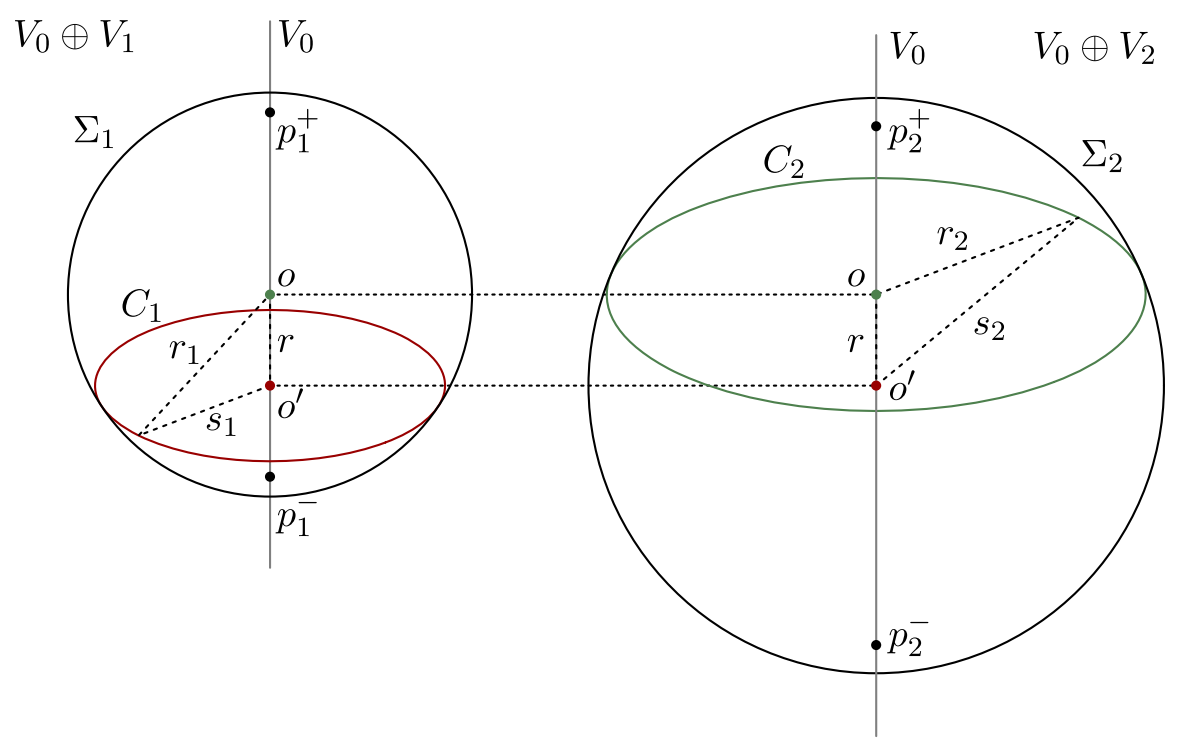

Fig. 4 Spheres $\Sigma_{i}$ and circles $C_{i}$ of a weak Lenz configuration in $\mathbb{R}^{5}$

a translate of a finite subset of $\Sigma_{1} \cup C_{2}$ a strong Lenz configuration (equivalent to the 5-dimensional "Lenz configuration" of Sect. 2).

To define a weak Lenz configuration takes more care than for odd $d \geq 7$. Choose an additional parameter $r \in\left[0, r_{1}\right)$ and a point $o^{\prime} \in V_{0}$ at distance $r$ to $o$. Let $C_{1}$ be the circle with center $o^{\prime}$ and radius $s_{1}:=\sqrt{r_{1}^{2}-r^{2}}$ in the plane of $V_{0} \oplus V_{1}$ parallel to $V_{1}$ that passes through $o^{\prime}$. Let $\Sigma_{2}$ be the 2-sphere in $V_{0} \oplus V_{2}$ with center $o^{\prime}$ and radius $s_{2}:=\sqrt{r_{2}^{2}+r^{2}}$. Then $C_{i} \subset \Sigma_{i}(i=1,2)$ (Fig. 4). Note that $s_{1}^{2}+s_{2}^{2}=1$, hence any point of $\Sigma_{2}$ and any point of $C_{1}$ are at unit distance. Similarly to Lemma 13, no point of $\Sigma_{1} \backslash C_{1}$ can be at unit distance to a point of $\Sigma_{2} \backslash C_{2}$. We call a translate of a finite subset of $\Sigma_{1} \cup \Sigma_{2}$ a weak Lenz configuration. As before, strong Lenz configurations are clearly weak. Assume without loss of generality that the weak Lenz configuration $S \subset \Sigma_{1} \cup \Sigma_{2}$. Each $\Sigma_{i}$ has two poles: $\left\{p_{1}^{+}, p_{1}^{-}\right\}:=V_{0} \cap \Sigma_{1}$ and $\left\{p_{2}^{+}, p_{2}^{-}\right\}:=V_{0} \cap \Sigma_{2}$. In general, $\Sigma_{1}$ and $\Sigma_{2}$ may not have a point in common. If they do, the common points will be coinciding poles. Define $S_{0}:=S \cap V_{0}, S_{i}:=S \cap \Sigma_{i} \backslash V_{0}(i=1,2)$, $n_{i}:=\left|S_{i}\right|(i=0,1,2)$, and $n:=|S|$.

\subsubsection{Unit Distances}

As for odd $d \geq 7$, define

$$
u_{5}^{L}(n)=\max \left\{u(S): S \text { is a weak Lenz configuration of } n \text { points in } \mathbb{R}^{5}\right\} .
$$

We call any weak Lenz configuration $S$ of $n$ points in $\mathbb{R}^{5}$ satisfying $u(S)=u_{5}^{L}(n)$ an optimized Lenz configuration (for unit distances). Again the best known estimate is $u_{5}^{L}(n)=t_{2}(n)+\Theta\left(n^{4 / 3}\right)$, due to Erdős and Pach [14]. We show that an optimized 
Lenz configuration is strong for sufficiently large $n$. As before, this implies that $u_{5}^{L}(n)$ can be determined if the function $g(n)$, which gives the maximum number of unit distances for $n$ points on a 2 -sphere of arbitrary radius, is known.

Proposition 16 For all sufficiently large n, all optimized Lenz configurations for unit distances on $n$ points in $\mathbb{R}^{5}$ are strong Lenz configurations.

Proof Let $S$ be an optimized Lenz configuration on $n$ points. Suppose that $S_{1} \backslash C_{1} \neq$ $\emptyset$ and $S_{2} \backslash C_{2} \neq \emptyset$. By Lemma 7(d), there exist points $x_{i} \in S_{i} \backslash C_{i}$ with $u\left(x_{i}, S_{i} \backslash C_{i}\right)=$ $O\left(n^{1 / 3}\right)(i=1,2)$. Since $x_{i} \notin S_{0}, u\left(x_{i}, C_{i}\right) \leq 2$. Thus $u\left(x_{i}, S_{i}\right)=O\left(n^{1 / 3}\right)$. If we replace each $x_{i}$ by a new point on $C_{i}$, we lose at most $O\left(n^{1 / 3}\right)$ unit distances and gain $\left|S_{1} \backslash C_{1}\right|+\left|S_{2} \backslash C_{2}\right|$. Since $S$ is extremal, $\left|S_{1} \backslash C_{1}\right|+\left|S_{2} \backslash C_{2}\right|=O\left(n^{1 / 3}\right)$. We bound $u(S)$ from above as in the case of odd $d \geq 7$. For each $i=1,2$ :

$$
\begin{aligned}
u\left(S_{i}\right) & \leq u\left(S_{i} \cup S_{0}\right) \\
& =u\left(S_{0}\right)+u\left(S_{0}, S_{i}\right)+u\left(S_{i} \cap C_{i}\right)+u\left(S_{i} \cap C_{i}, S_{i} \backslash C_{i}\right)+u\left(S_{i} \backslash C_{i}\right) \\
& \leq 4+4\left|S_{i} \cap C_{i}\right|+\left|S_{i} \cap C_{i}\right|+2\left|S_{i} \backslash C_{i}\right|+O\left(\left|S_{i} \backslash C_{i}\right|^{4 / 3}\right) \\
& =O(n),
\end{aligned}
$$

hence,

$$
\begin{aligned}
u(S) & =u\left(S_{1}, S_{2}\right)+u\left(S_{0} \cup S_{1}\right)+u\left(S_{0} \cup S_{2}\right)+u\left(S_{0}\right)+u\left(S_{1}\right)+u\left(S_{2}\right) \\
& \leq t_{2}(n)+O(n),
\end{aligned}
$$

which contradicts $u(S)=t_{2}(n)+\Theta\left(n^{4 / 3}\right)$.

Therefore, some $S_{i} \backslash C_{i}=\emptyset$; without loss of generality, $S_{2} \backslash C_{2}=\emptyset$. To show that $S$ is a strong Lenz configuration, it remains to show that $S_{0} \subset \Sigma_{1}$. Suppose then without loss of generality that $p_{2}^{+} \in S_{0}$ and $p_{2}^{+} \notin \Sigma_{1}$. Then $p_{1}^{ \pm} \neq p_{2}^{+}$. Since $p_{1}^{+}$is at unit distance to each point of $C_{2}$, and $p_{1}^{+}$and $p_{2}^{+}$are different points in $V_{0}$, it follows that $p_{2}^{+}$is not at unit distance to any point in $S_{2}$. If we replace $p_{2}^{+}$by a new point on $C_{2}$, we lose at most one unit distance (possibly between $p_{2}^{+}$and $p_{2}^{-}$) and gain $\left|S \cap \Sigma_{1} \backslash C_{1}\right|$ unit distances. By extremality, $\left|S \cap \Sigma_{1} \backslash C_{1}\right| \leq 1$. Therefore, except for at most 3 points (in addition, $p_{2}^{+} \in S_{0}$ and possibly $p_{2}^{-} \in S_{0}$ ), $S$ is on two orthogonal circles, and for this essentially 4-dimensional configuration, we obtain $u(S) \leq t_{2}(n)+O(n)$ as before, a contradiction.

It follows that $S$ is a strong Lenz configuration.

\subsubsection{Diameters}

As for odd $d \geq 7$, define

$$
M_{5}^{L}(n)=\max \left\{u(S): S \text { is a weak Lenz configuration of } n \text { points in } \mathbb{R}^{5}\right\} .
$$

We call any diameter 1 weak Lenz configuration $S$ of $n$ points in $\mathbb{R}^{5}$ satisfying $M(S)=u(S)=M_{5}^{L}(n)$ an optimized Lenz configuration (for diameters). Again an 
optimized Lenz configuration is strong for large $n$, and the exact value of $M_{5}^{L}(n)$ can be determined. This is more intricate than for odd $d \geq 7$.

Proposition 17 For all sufficiently large n, all optimized Lenz configurations for diameters on $n$ points in $\mathbb{R}^{5}$ are strong Lenz configurations. Furthermore, $M_{5}^{L}(n)=$ $t_{2}(n)+n$.

Proof We first describe two types of strong Lenz configurations on $n$ points with $t_{2}(n)+n$ diameters.

In the first construction, choose $r_{1}$ such that there exists a set $S_{1}$ of $n_{1}$ points of diameter 1 on $\Sigma_{1}$ with $2 n_{1}-2$ diameters. By Lemma 7(e) this is possible if $n_{1} \geq 4$, $n_{1} \neq 5$. Choose any set $S_{2}$ of $n_{2}=n-n_{1}$ points of diameter 1 on $C_{2}$. (Note that $r_{1}<1 / \sqrt{2}$ by Lemma 7(f), which gives $r_{2}>1 / \sqrt{2}>1 / \sqrt{3}$. Then by Lemma 7(c), we can have at most one diameter of length 1 on $C_{2}$.) Let $S:=S_{1} \cup S_{2}$. Then

$$
\begin{aligned}
u(S) & =u\left(S_{1}, S_{2}\right)+u\left(S_{1}\right)+u\left(S_{2}\right) \\
& =n_{1} n_{2}+2 n_{1}-2+1=n_{1}\left(n_{2}+2\right)-1 \\
& \leq t_{2}(n+2)-1=t_{2}(n)+n .
\end{aligned}
$$

Equality is possible by taking $n_{1}=\lfloor n / 2\rfloor+1$ or $\lceil n / 2\rceil+1$. Keeping in mind that $n_{1} \geq 4, n_{1} \neq 5$, we obtain $t_{2}(n)+n$ diameters for all $n \geq 6, n \neq 8$.

In the second construction, first choose $r_{2}$ such that there exists a set $S_{2}$ of $n_{2}$ points of diameter 1 on $C_{2}$ with $n_{2}$ diameters (a regular star polygon). By Lemma 7(b) this is possible if $n_{2} \geq 3$ is odd. Then $r_{2} \leq 1 / \sqrt{3}$ by Lemma 7(c), and $r_{1} \geq \sqrt{2 / 3}>$ $1 / \sqrt{2}$. By Lemma 7 (f) we can then choose a set $S_{1}$ of $n_{1}=n-n_{2}$ points of diameter 1 on $\Sigma_{1}$ with $n_{1}$ diameters if $n_{1} \geq 3$. Let $S:=S_{1} \cup S_{2}$. Then

$$
\begin{aligned}
u(S) & =u\left(S_{1}, S_{2}\right)+u\left(S_{1}\right)+u\left(S_{2}\right) \\
& =n_{1} n_{2}+n_{1}+n_{2}=\left(n_{1}+1\right)\left(n_{2}+1\right)-1 \\
& \leq t_{2}(n+2)-1=t_{2}(n)+n .
\end{aligned}
$$

Equality is possible by taking $n_{1}=\lfloor n / 2\rfloor, n_{2}=\lceil n / 2\rceil$ or $n_{1}=\lceil n / 2\rceil, n_{2}=\lfloor n / 2\rfloor$. Keeping in mind the requirements that $n_{2} \geq 3$ must be odd and $n_{1} \geq 3$, we obtain $t_{2}(n)+n$ diameters for all $n \geq 6, n \neq 0(\bmod 4)$. (It is because this second, simpler construction does not work for all $n$ that we need the construction in Lemma 7(e) of an odd number $n_{1}$ of points on a 2 -sphere with $2 n_{1}-2$ diameters.)

Summarizing, $M_{5}^{L}(n) \geq t_{2}(n)+n$ for all $n \geq 9$. It is easy to see that every strong Lenz configuration with at least $t_{2}(n)+n$ diameters must be one of the above two constructions for sufficiently large $n$. We now turn to weak Lenz configurations.

Let $S$ be an optimized Lenz configuration on $n$ points. We distinguish between two cases.

First case: $S \cap \Sigma_{1} \cap \Sigma_{2} \neq \emptyset$. Any point in $S \cap \Sigma_{1} \cap \Sigma_{2}$ must be a common pole of $\Sigma_{1}$ and $\Sigma_{2}$, say $p_{1}^{+}=p_{2}^{+}$. Since this point is at distance 1 to $C_{1}$ and $C_{2}$, it follows that $\left|p_{1}^{+} p_{1}^{-}\right|,\left|p_{2}^{+} p_{2}^{-}\right|>1$. Therefore, $S \cap \Sigma_{1} \cap \Sigma_{2}$ contains only one point $p:=p_{1}^{+}=p_{2}^{+}$ 
at distance 1 to both $C_{1}$ and $C_{2}$. Let $k_{i}:=\left|S_{i} \backslash C_{i}\right|(i=1,2)$. Then

$$
\begin{aligned}
t_{2}(n)+n & \leq u(S) \\
& =u\left(S_{1}, S_{2}\right)+u\left(S_{1} \cup\{p\}\right)+u\left(S_{2} \cup\{p\}\right) \\
& =n_{1} n_{2}-k_{1} k_{2}+u\left(S_{1} \cup\{p\}\right)+u\left(S_{2} \cup\{p\}\right) .
\end{aligned}
$$

If $u\left(S_{i} \cup\{p\}\right) \leq n_{i}+1$ for both $i=1,2$, then by substituting into (2),

$$
\begin{aligned}
t_{2}(n)+n & \leq n_{1} n_{2}-k_{1} k_{2}+n_{1}+1+n_{2}+1 \\
& =\left(n_{1}+1\right)\left(n_{2}+1\right)-k_{1} k_{2}+1 \\
& \leq t_{2}(n+1)-k_{1} k_{2}+1 \quad\left(\text { note } n_{1}+n_{2}+1=n\right) \\
& =t_{2}(n)+\lceil n / 2\rceil-k_{1} k_{2}+1 .
\end{aligned}
$$

Therefore, $\lfloor n / 2\rfloor+k_{1} k_{2} \leq 1$, a contradiction.

Without loss of generality, we may therefore assume that $u\left(S_{1} \cup\{p\}\right)>n_{1}+1$. By Lemma 7(f), $r_{1}<1 / \sqrt{2}$, which gives $r_{2}>1 / \sqrt{2}$ and $u\left(S_{2} \cup\{p\}\right) \leq n_{2}+1$ (again Lemma 7(f)). Also, $u\left(S_{1} \cup\{p\}\right) \leq 2\left(n_{1}+1\right)-2=2 n_{1}$ (Lemma 7(e)). Substituting into (2), we have

$$
\begin{aligned}
t_{2}(n)+n & \leq n_{1} n_{2}-k_{1} k_{2}+2 n_{1}+n_{2}+1 \\
& =\left(n_{1}+1\right)\left(n_{2}+2\right)-k_{1} k_{2}-1 \\
& \leq t_{2}(n+2)-k_{1} k_{2}-1 \\
& =t_{2}(n)+n-k_{1} k_{2} .
\end{aligned}
$$

It follows that $k_{1} k_{2}=0, S$ is a strong Lenz configuration, and $u(S)=t_{2}(n)+n$.

Second case: $S \cap \Sigma_{1} \cap \Sigma_{2}=\emptyset$. Then $S$ may still contain poles, but a pole of $\Sigma_{i}$ in $S$ is not at distance 1 to $C_{i}$ (otherwise it would also be a pole of the other sphere). We now define $T_{i}=S \cap \Sigma_{i}(i=1,2)$. Then $T_{1}, T_{2}$ partition $S$ (and we forget about the partition $\left.S_{0}, S_{1}, S_{2}\right)$. Let $m_{i}:=\left|T_{i}\right|$ and $k_{i}:=\left|T_{i} \backslash C_{i}\right|(i=1,2)$. As in the first case,

$$
\begin{aligned}
t_{2}(n)+n & \leq u(S) \\
& =u\left(T_{1}, T_{2}\right)+u\left(T_{1}\right)+u\left(T_{2}\right) \\
& =m_{1} m_{2}-k_{1} k_{2}+u\left(T_{1}\right)+u\left(T_{2}\right) .
\end{aligned}
$$

If $u\left(T_{i}\right) \leq m_{i}$ for both $i=1,2$, then by substituting into (3),

$$
\begin{aligned}
t_{2}(n)+n & \leq m_{1} m_{2}-k_{1} k_{2}+m_{1}+m_{2} \\
& =\left(m_{1}+1\right)\left(m_{2}+1\right)-k_{1} k_{2}-1 \\
& \leq t_{2}(n+2)-k_{1} k_{2}-1 \\
& =t_{2}(n)+n-k_{1} k_{2} .
\end{aligned}
$$


It follows that $k_{1} k_{2}=0, S$ is a strong Lenz configuration, and $u(S)=t_{2}(n)+n$.

Otherwise, without loss of generality, $u\left(T_{1}\right)>m_{1}$. As in the first case,

$$
u\left(T_{1}\right) \leq 2 m_{1}-2
$$

and

$$
u\left(T_{2}\right) \leq m_{2}
$$

Since each point in $T_{i} \backslash C_{i}$ is at distance 1 to at most two points of $T_{i} \cap C_{i}$ (recall that in this case a pole is not at distance 1 to any point on $C_{i}$ ), we also obtain

$$
\begin{aligned}
u\left(T_{1}\right) & =u\left(T_{1} \cap C_{1}\right)+u\left(T_{1} \cap C_{1}, T_{1} \backslash C_{1}\right)+u\left(T_{1} \backslash C_{1}\right) \\
& \leq\left|T_{1} \cap C_{1}\right|+2\left|T_{1} \backslash C_{1}\right|+2\left|T_{1} \backslash C_{1}\right|-2 \\
& =m_{1}+3 k_{1}-2
\end{aligned}
$$

and, since $r_{2}>1 / \sqrt{2}$,

$$
\begin{aligned}
u\left(T_{2}\right) & =u\left(T_{2} \cap C_{2}\right)+u\left(T_{2} \cap C_{2}, T_{2} \backslash C_{2}\right)+u\left(T_{2} \backslash C_{2}\right) \\
& \leq 1+2\left|T_{2} \backslash C_{2}\right|+\left|T_{2} \backslash C_{2}\right| \\
& =1+3 k_{2} .
\end{aligned}
$$

Substituting (5) and (6) into (3), we have

$$
\begin{aligned}
t_{2}(n)+n & \leq m_{1} m_{2}-k_{1} k_{2}+m_{1}+3 k_{1}-2+m_{2} \\
& =\left(m_{1}+1\right)\left(m_{2}+1\right)-k_{1}\left(k_{2}-3\right)-3 \\
& \leq t_{2}(n+2)-k_{1}\left(k_{2}-3\right)-3 \\
& =t_{2}(n)+n-k_{1}\left(k_{2}-3\right)-2 .
\end{aligned}
$$

Therefore, $k_{1}\left(k_{2}-3\right)+2 \leq 0$, hence $k_{2} \leq 2$.

Substituting (4) and (7) into (3), we have

$$
\begin{aligned}
t_{2}(n)+n & \leq m_{1} m_{2}-k_{1} k_{2}+2 m_{1}-2+3 k_{2}+1 \\
& =m_{1}\left(m_{2}+2\right)-\left(k_{1}-3\right) k_{2}-1 \\
& \leq t_{2}(n+2)-\left(k_{1}-3\right) k_{2}-1 \\
& =t_{2}(n)+n-\left(k_{1}-3\right) k_{2} .
\end{aligned}
$$

Therefore, $\left(k_{1}-3\right) k_{2} \leq 0$. If $k_{2}>0$, then $k_{1} \leq 3$, and substituting (6) and (7) into (3),

$$
\begin{aligned}
t_{2}(n)+n & \leq m_{1} m_{2}-k_{1} k_{2}+m_{1}+3 k_{1}-2+3 k_{2}+1 \\
& =m_{1}\left(m_{2}+1\right)+O(1) \\
& \leq t_{2}(n+1)+O(1) \\
& =t_{2}(n)+\lceil n / 2\rceil+O(1),
\end{aligned}
$$


a contradiction. It follows that $k_{2}=0$, hence $S$ is a strong Lenz configuration, and $u(S)=t_{2}(n)+n$.

\section{Stability Theorems}

We formulate the stability theorem of Erdős [8] and Simonovits [26] (see also [2, Chap. 5, Theorem 4.2]) in the following convenient way. Let $K_{r}(t)$ denote the complete $r$-partite graph with $t$ vertices in each class.

Stability Theorem For any $p, t \geq 2$ and any $\varepsilon>0$, there exist $N$ and $\delta>0$ such that if $G$ is any graph with $n \geq N$ vertices and at least $\left(\frac{p-1}{2 p}-\delta\right) n^{2}$ edges which does not contain $K_{p+1}(t)$, then the vertices of $G$ can be partitioned into sets $S_{0}, S_{1}, \ldots, S_{p}$ such that $\left|S_{0}\right|<\varepsilon n$,

$$
\frac{n}{p}-\varepsilon n<\left|S_{i}\right|<\frac{n}{p}+\varepsilon n \quad \text { for each } i=1, \ldots, p,
$$

and for each $i=1, \ldots, p$, each $x \in S_{i}$ is nonadjacent to less than $\varepsilon$ n vertices of $G-S_{i}$.

We now use the Stability Theorem to prove Theorems 4 and 5.

Proof of Theorem 4 Without loss of generality, $\varepsilon<1 /\left(3 p^{2}\right)$. By Lemma 8 (b), $K_{p+1}(3)$ does not occur in the unit distance graph of $S$. Let $S_{0}, S_{1}, \ldots, S_{p}$ be the partition coming from the Stability Theorem. Suppose that $S_{1}$ does not lie on any circle. Let $A_{1}$ be a set of 4 nonconcyclic points of $S_{1}$. For each $i=2, \ldots, p$, let $A_{i}$ consist of 3 points of $S_{i}$ such that any two vertices in distinct $A_{i}$ 's are adjacent. This is possible, since each $x \in S_{i}$ is at unit distance to all points in $S \backslash S_{i}$ except for $\varepsilon n$ points, and $(4+3(p-2)) \varepsilon n+3<n / p-\varepsilon n$ if $n>9 p^{2}$. The unit distance graph of $\bigcup_{i=1}^{p} A_{i}$ contains a complete $p$-partite graph with 4 vertices in the one class $A_{1}$ and 3 vertices in each other class $A_{i}, i=2, \ldots, p$. By Lemma 8(a), each $A_{i}$ is concyclic, a contradiction.

Therefore, each $S_{i}(i=1, \ldots, p)$ is concyclic. To see that these circles are orthogonal, choose 3 points from each $S_{i}$ as above to form a $K_{p}(3)$. Again by Lemma 8(a) each class lies on a circle $C_{i}$, with $C_{1}, \ldots, C_{p}$ mutually orthogonal. Since there is a unique circle through any 3 noncollinear points, $S_{i} \subset C_{i}$ for each $i=1, \ldots, p$.

The following is the even-dimensional case of Corollary 6.

Corollary 18 Fix an even $d \geq 4$. If a set $S$ of $n$ points in $\mathbb{R}^{d}$ has at least $\left(\frac{p-1}{2 p}-\right.$ $o(1)) n^{2}$ unit distance pairs, then $S$ is a Lenz configuration except for $o(n)$ points.

Proof of Theorem 5 Without loss of generality, $\varepsilon<1 /\left(4 p^{2}\right)$. By Lemma 8(b), $K_{p+1}$ (3) does not occur in the unit distance graph of $S$. Let $S_{0}, S_{1}, \ldots, S_{p}$ be the partition coming from the Stability Theorem using $\varepsilon^{\prime}=\varepsilon / 5$. Suppose that $S_{1}$ does not lie on any 2 -sphere. Let $A_{1}$ be a set of 5 noncospherical points of $S_{1}$. For each 
$i=2, \ldots, p$, let $A_{i}$ consist of 3 points of $S_{i}$ such that any two vertices in distinct $A_{i}$ 's are adjacent. This is possible, since each $x \in S_{i}$ is at unit distance to all points in $S \backslash S_{i}$ except for $\varepsilon^{\prime} n$ points, and $(5+3(p-2)) \varepsilon^{\prime} n+3<n / p-\varepsilon^{\prime} n$ if $n>4 p$. The unit distance graph of $\bigcup_{i=1}^{p} A_{i}$ contains a complete $p$-partite graph with 5 vertices in one class and 3 vertices in each other class. By Lemma 8(a), each $A_{i}$ is on a 2-sphere, a contradiction.

Therefore, each $S_{i}(i=1, \ldots, p)$ is on a 2 -sphere. If each $S_{i}$ lies on a circle, then, as in the even-dimensional case, it follows that these circles are orthogonal, and the proof is finished. Without loss of generality $S_{1}$ is then not concyclic. Let $\Sigma_{1}$ denote the unique 2-sphere on which $S_{1}$ lies. Let $A_{1}$ be a set of 4 noncoplanar points of $S_{1}$.

We now slightly modify the partition of $S$. There are less than $4 \varepsilon^{\prime} n$ points of $\bigcup_{i=2}^{p} S_{i}$ not at distance 1 to each point of $A_{1}$. Remove these points from $\bigcup_{i=2}^{p} S_{i}$ and add them to $S_{0}$. Thus we may assume that each point of $A_{1}$ is joined to all of $\bigcup_{i=2}^{p} S_{i}$, but then $\left|S_{0}\right|<5 \varepsilon^{\prime} n=\varepsilon n$ for each $i=1, \ldots, p,|| S_{i}|-n / p|<\varepsilon n$, and each point of $S_{i}$ is joined to less than $\varepsilon n$ points of $S \backslash S_{i}$. We show that for this modified partition, $S_{2}, \ldots, S_{p}$ are on circles $C_{2}, \ldots, C_{p}$, with $\Sigma_{1}, C_{2}, \ldots, C_{p}$ mutually orthogonal.

Suppose that some $S_{i}(i=2, \ldots, p)$ is not concyclic, without loss of generality $S_{2}$. Let $A_{2}$ be a set of 4 nonconcyclic points from $S_{2}$, and, as before, for $i=3, \ldots, p$, let $A_{i}$ consist of 3 points from $S_{i}$ such that all vertices in different $A_{i}$ 's are adjacent. By Lemma $8\left(\right.$ a), all $A_{i}, i=2, \ldots, p$, must lie on circles, a contradiction.

Therefore, each $S_{i}(i=2, \ldots, p)$ is on a circle $C_{i}$. As in the proof of Theorem 4 , to see that $\Sigma_{1}, C_{2}, \ldots, C_{p}$ are mutually orthogonal, choose 4 noncoplanar points from $S_{1}$ and 3 points from the other $S_{i}$ that form a complete $p$-partite graph, and apply Lemma 8(a).

The following is the odd-dimensional case of Corollary 6.

Corollary 19 Fix an odd $d \geq 5$. If a set $S$ of $n$ points in $\mathbb{R}^{d}$ has at least $\left(\frac{p-1}{2 p}-\right.$ $o(1)) n^{2}$ unit distance pairs, then $S$ is a strong Lenz configuration except for $o(n)$ points.

\section{Extremal Sets Are (Weak) Lenz Configurations}

The following three propositions, completing the proof of the main theorem, now follow relatively simply from the stability theorems.

Proposition 20 For each even $d \geq 4$, there exists $N(d)$ such that all sets of $n \geq$ $N(d)$ points in $\mathbb{R}^{d}$ extremal with respect to unit distances or diameters are Lenz configurations.

Proof When considering diameters assume that the diameter is 1 . In both cases, an extremal set $S$ on $n$ points has at least $t_{p}(n)=\frac{p-1}{2 p} n^{2}-O(1)$ unit distances, so we may apply Theorem 4 (with $\varepsilon=1 /\left(2 p^{2}\right)$ ). Thus, for $n$ sufficiently large depending on $d$, there is a partition $S_{0}, S_{1}, \ldots, S_{p}$ of $S$ with $\left|S_{0}\right|<\varepsilon n$ and for $i=1, \ldots, p$, ||$S_{i}|-n / p|<\varepsilon n$ and the $S_{i}$ are on orthogonal circles $C_{i}$. 
We use the extremality of $S$ to show that $S_{0} \subset \bigcup_{i=1}^{p} C_{i}$. Let $x \in S_{0}$. If $u\left(x, S_{i}\right) \geq 3$ for all $i=2, \ldots, p$, then by Lemma $8(\mathrm{c}), x \in C_{1}$. Thus, without loss of generality, $u\left(x, S_{i}\right) \leq 2$ for at least two $i$ 's, say $i=1,2$. Then

$$
\begin{aligned}
u(x, S) & =\sum_{i=0}^{p} u\left(x, S_{i}\right) \leq\left|S_{0}\right|-1+2+2+\sum_{i=3}^{p}\left|S_{i}\right| \\
& <\varepsilon n-1+4+(p-2)\left(\frac{n}{p}+\varepsilon n\right)=\left(1-\frac{2}{p}+\varepsilon(p-1)\right) n+3 .
\end{aligned}
$$

If we remove $x$ and replace it with a new point $x^{\prime} \in C_{1}$, then

$$
\begin{aligned}
u\left(x^{\prime}, S \backslash\{x\}\right) & \geq u\left(x^{\prime}, \bigcup_{i=2}^{p} S_{i}\right)=\sum_{i=2}^{p}\left|S_{i}\right| \\
& >(p-1)\left(\frac{n}{p}-\varepsilon n\right)=\left(1-\frac{1}{p}-(p-1) \varepsilon\right) n .
\end{aligned}
$$

In the case of diameters, we have to take care that $x^{\prime}$ does not increase the diameter. This can be done as follows.

Since all points of $C_{1}$ are already at unit distance to all points of $\bigcup_{i=2}^{p} C_{i}$, it is sufficient to choose $x^{\prime}$ at distance at most 1 to each point of $S_{0}$. When $d \geq 6, C_{1}$ has radius $1 / \sqrt{2}$, hence $S_{1}$ is contained in a $90^{\circ}$ arc $\gamma$ of $C_{1}$. The set of points on $C_{1}$ at distance larger than 1 from some $y \in S_{0}$ is a (perhaps empty) subarc of $\gamma$. Such a subarc does not contain any point of $S_{1}$ and is therefore between some two consecutive points of $S_{1}$. Since $\left|S_{1}\right| \geq\left|S_{0}\right|+1$ for $n$ sufficiently large, there exist two consecutive points of $S_{1}$, say $a$ and $b$, with no subarc between them. Therefore, all points on $C_{1}$ between $a$ and $b$ are at distance at most 1 to all points of $S_{0}$, and we may choose $x^{\prime}$ to be any point on $C_{1}$ between $a$ and $b$. When $d=4$, one of the two circles $C_{1}$ and $C_{2}$ has radius at least $1 / \sqrt{2}$, and the above argument also works for this circle.

Since $S$ is extremal, such a modification cannot increase the number of unit distances. Thus, $u(S) \geq u\left(S \cup\left\{x^{\prime}\right\} \backslash\{x\}\right)$, hence $u(x, S) \geq u\left(x^{\prime}, S \backslash\{x\}\right)$, i.e.,

$$
\left(1-\frac{2}{p}+\varepsilon(p-1)\right) n+3>\left(1-\frac{1}{p}-\varepsilon(p-1)\right) n,
$$

which is a contradiction if $\varepsilon=1 /\left(2 p^{2}\right)$ and $n \geq 3 p^{2}$.

We have shown that $S_{0} \subset \bigcup_{i=1}^{p} C_{i}$ for large $n$, which implies that $S$ is a Lenz configuration.

Proposition 21 For each odd $d \geq 7$, there exists $N(d)$ such that all sets of $n \geq$ $N(d)$ points in $\mathbb{R}^{d}$ extremal with respect to unit distances or diameters are weak Lenz configurations.

Proof Again in the case of diameters, assume that the diameter is 1. Again we apply Theorem 5 (with $\varepsilon=1 /\left(4 p^{2}\right)$ ). Thus, for $n$ sufficiently large depending on $d$, there is 
a partition $S_{0}, S_{1}, \ldots, S_{p}$ of $S$ with $\left|S_{0}\right|<\varepsilon n$ and for $i=1, \ldots, p,|| S_{i}|-n / p|<\varepsilon n$, $S_{1}$ is on a sphere $\Sigma_{1}$, each $S_{i}(i=2, \ldots, p)$ is on a circle $C_{i}$, and $\Sigma_{1}, C_{2}, \ldots, C_{p}$ are mutually orthogonal and all have radius $1 / \sqrt{2}$.

To show that $S$ is a weak Lenz configuration, it is sufficient to show that each point of $S_{0}$ not on $\Sigma_{1}$ lies on the 2 -sphere of radius $1 / \sqrt{2}$ containing some $C_{i}$ ( $i=$ $2, \ldots, p)$ in the subspace generated by $C_{i}$ and some fixed diameter of $\Sigma_{1}$.

As in the proof of Proposition 20, there exists a point $x^{\prime} \in C_{2}$ that does not increase the diameter. Since $S$ is extremal, for any $x \in S$,

$$
\begin{aligned}
u(x, S) & \geq u\left(x^{\prime}, S \backslash\{x\}\right) \geq \sum_{\substack{i=1 \\
i \neq 2}}^{p}\left|S_{i}\right| \\
& >(p-1)\left(\frac{n}{p}-\varepsilon n\right)=\left(1-\frac{1}{p}-(p-1) \varepsilon\right) n .
\end{aligned}
$$

For $i=2, \ldots, p$, define

$$
T_{i}:=\left\{x \in S_{0}: u\left(x, S_{i}\right) \leq 2\right\} .
$$

For any point $x \in \Sigma_{1}, u\left(x, S_{i}\right)=\left|S_{i}\right|>\frac{n}{p}-\varepsilon n \geq 3$ for $n>4 p$, and therefore $\bigcup_{i=2}^{p} T_{i} \subseteq S_{0} \backslash \Sigma_{1}$. Conversely, if $x \in S_{0}$ and $u\left(x, S_{i}\right) \geq 3$ for each $i=2, \ldots, p$, then $x \in \Sigma_{1}$ (Lemma 8(c)). It follows that $\bigcup_{i=2}^{p} T_{i}=S_{0} \backslash \Sigma_{1}$. We next show that $T_{2}, \ldots, T_{p}$ partition $S_{0} \backslash \Sigma_{1}$. If not, there exists $x \in S_{0} \backslash \Sigma_{1}$ with $u\left(x, S_{i}\right) \leq 2$ and $u\left(x, S_{j}\right) \leq 2$ for distinct $i, j \in\{2, \ldots, p\}$. Then

$$
\begin{aligned}
u(x, S) & =u\left(x, S_{0}\right)+u\left(x, S_{1}\right)+\sum_{i=2}^{p} u\left(x, S_{i}\right) \\
& <\varepsilon n+\left(\frac{n}{p}+\varepsilon n\right)+2+2+(p-3)\left(\frac{n}{p}+\varepsilon n\right) \\
& =\left(1-\frac{2}{p}+(p-1) \varepsilon\right) n+4,
\end{aligned}
$$

which contradicts the lower bound (8) when $n>8 p$.

Note that the neighbors in $S_{1}$ of an $x \in S_{0} \backslash \Sigma_{1}$ all lie on a circle $C_{x}$, say, on $\Sigma_{1}$. We now show that $C_{x}$ is the same circle for all $x \in S_{0} \backslash \Sigma_{1}$. First we bound $u\left(x, S_{1}\right)$ from below:

$$
\begin{aligned}
u(x, S) & =u\left(x, S_{0}\right)+u\left(x, S_{1}\right)+\sum_{i=2}^{p} u\left(x, S_{i}\right) \\
& <\varepsilon n+u\left(x, S_{1}\right)+2+(p-2)\left(\frac{n}{p}+\varepsilon n\right) \\
& =u\left(x, S_{1}\right)+\left(1-\frac{2}{p}+(p-1) \varepsilon\right) n+2,
\end{aligned}
$$


which, together with estimate (8), gives

$$
u\left(x, S_{1}\right)>\left(\frac{1}{p}-2(p-1) \varepsilon\right) n-2 .
$$

If $C_{y} \neq C_{x}$ for some $y \in S_{0} \backslash \Sigma_{1}$, then

$$
\left|S_{1}\right| \geq u\left(x, S_{1}\right)+u\left(y, S_{1}\right)-2>2\left(\frac{1}{p}-2(p-1) \varepsilon\right) n-6 .
$$

This contradicts $\left|S_{1}\right|<\frac{n}{p}+\varepsilon n$ when $n>8 p^{2}$.

Therefore, the neighbors in $S_{1}$ of any $x \in S_{0} \backslash \Sigma_{1}$ are on the same circle $C_{x}=: C$. For each $i=2, \ldots, p$, let $\Sigma_{i}$ be the sphere of radius $1 / \sqrt{2}$ which has $C_{i}$ as great circle in the 3-space containing $C_{i}$ and the diameter of $\Sigma_{1}$ perpendicular to $C$. Since $T_{2}, \ldots, T_{p}$ form a partition of $S_{0} \backslash \Sigma_{1}$, each point of $T_{i}$ is at distance 1 to at least 3 points of each $C_{j}, j \neq i$. By (9), each point of $S_{0} \backslash \Sigma_{1}$ also has at least 3 neighbors on $C$ if $n \geq 10 p$. By Lemma 8(d) it follows that $T_{i} \subset \Sigma_{i}$. Since also $S_{i} \subset \Sigma_{i}$, we obtain that $S$ is a weak Lenz configuration for large $n$.

Proposition 22 For all sufficiently large $n$, all sets of $n$ points in $\mathbb{R}^{5}$ extremal with respect to unit distances or diameters are weak Lenz configurations.

Proof An extremal set $S$ of $n$ points has at least $n^{2} / 4$ unit distances, so by Theorem 5 with $\varepsilon=1 / 11$ we obtain that for sufficiently large $n, S$ can be partitioned into $S_{0}, S_{1}, S_{2}$ such that $\left|S_{0}\right|<\varepsilon n,|| S_{i}|-n / 2|<\varepsilon n(i=1,2), S_{1}$ is on a sphere $\Sigma_{1}$ of radius $r_{1}, S_{2}$ is on a circle $C_{2}$ of radius $r_{2}$, with $\Sigma_{1}$ and $C_{2}$ orthogonal, and $r_{1}^{2}+r_{2}^{2}=1$.

As in the proof for odd $d \geq 7$, if $r_{2} \geq 1 / \sqrt{2}$, we can find a point $x^{\prime} \in C_{2}$ that does not increase the diameter. Otherwise, $r_{1} \geq 1 / \sqrt{2}$, and we consider the intersection of $\Sigma_{1}$ and all balls in the 3 -space of $\Sigma_{1}$ of radius 1 centered at points in $S \cap \Sigma_{1}$. This gives a spherically convex set on $\Sigma_{1}$ containing $S \cap \Sigma_{1}$. Any new point $x^{\prime}$ in this set is at distance at most 1 to all points of $S$. As before, replacing any point $x \in S$ by $x^{\prime}$ gives $u(x, S)>\left(\frac{1}{2}-\varepsilon\right) n$. Note that if $u\left(x, S_{2}\right) \geq 3$ for some $x \in S_{0}$, then $x \in \Sigma_{1}$. Therefore, $u\left(x, S_{2}\right) \leq 2$ for all $x \in S_{0} \backslash \Sigma_{1}$. Next we bound $u\left(x, S_{1}\right)$ from below for all $x \in S_{0} \backslash \Sigma_{1}$ :

$$
\begin{aligned}
\left(\frac{1}{2}-\varepsilon\right) n & <u(x, S)=u\left(x, S_{0}\right)+u\left(x, S_{1}\right)+u\left(x, S_{2}\right) \\
& <\varepsilon n+u\left(x, S_{1}\right)+2
\end{aligned}
$$

hence

$$
u\left(x, S_{1}\right)>\left(\frac{1}{2}-2 \varepsilon\right) n-2 .
$$

The neighbors in $S_{1}$ of an $x \in S_{0} \backslash \Sigma_{1}$ lie on a circle $C_{1}$, say, of $\Sigma_{1}$. If the neighbors of some other $y \in S_{0} \backslash \Sigma_{1}$ lie on another circle of $\Sigma_{1}$, then 


$$
\begin{aligned}
\frac{n}{2}+\varepsilon n & >\left|S_{1}\right|>u\left(x, S_{1}\right)+u\left(y, S_{1}\right)-2 \\
& >(1-4 \varepsilon) n-6 .
\end{aligned}
$$

Therefore, $5 \varepsilon n>n / 2-6$, a contradiction for $n$ sufficiently large. It follows that all neighbors in $S_{1}$ of any $y \in S_{0} \backslash \Sigma_{1}$ are on $C_{1}$. Let the radius of $C_{1}$ be $s_{1}$. By Lemma 8(c), each $x \in S_{0} \backslash \Sigma_{1}$ lies on the complementary sphere $\Sigma_{2}$ of radius $s_{2}$, where $s_{1}^{2}+s_{2}^{2}=1$, and $C_{2} \subset \Sigma_{2}$. We have shown that $S$ is a weak Lenz configuration for large $n$.

Acknowledgements I thank Peter Brass and János Pach for their encouragement and the anonymous referees for their careful reading.

\section{References}

1. Brass, P.: On the maximum number of unit distances among $n$ points in dimension four. In: Bárány, I., et al. (eds.) Intuitive Geometry. Bolyai Soc. Mathematical Studies, vol. 6, pp. 277-290. Jànos Bolyai Math. Soc., Budapest (1997). See also the review of this paper in Mathematical Reviews MR 98j:52030

2. Bollobás, B.: Extremal Graph Theory. Academic Press, London (1978). Reprinted by Dover, Mineola (2004)

3. Clarkson, K., Edelsbrunner, H., Guibas, L., Sharir, M., Welzl, E.: Combinatorial complexity bounds for arrangements of curves and surfaces. Discrete Comput. Geom. 5, 99-160 (1990)

4. Erdős, P.: On sets of distances of $n$ points. Am. Math. Mon. 53, 248-250 (1946)

5. Erdős, P.: On sets of distances of $n$ points in Euclidean space. Magy. Tud. Akad. Mat. Kut. Int. Közl. 5, 165-169 (1960)

6. Erdős, P.: Extremal problems in graph theory. In: Theory of Graphs and its Applications, Proc. Sympos. Smolenice, 1963, pp. 29-36. Publ. House Czech. Acad. Sci., Prague (1964)

7. Erdős, P.: On some applications of graph theory to geometry. Can. J. Math. 19, 968-971 (1967)

8. Erdős, P.: On some new inequalities concerning extremal properties of graphs. In: Erdôs, P., Katona, G. (eds.) Theory of Graphs, pp. 279-319. Academic Press, New York (1968)

9. Erdôs, P.: Combinatorial problems in geometry and number theory. In: Relations between combinatorics and other Parts of Mathematics, Ohio State Univ., Columbus, OH, 1978. Proc. Sympos. Pure Math., vol. XXXIV, pp. 149-162. American Mathematical Society, Providence (1979)

10. Erdős, P.: On some metric and combinatorial geometric problems. Discrete Math. 60, 147-153 (1986)

11. Erdős, P.: Some of my favourite unsolved problems. In: A Tribute to Paul Erdős, pp. 467-478. Cambridge University Press, Cambridge (1990)

12. Erdős, P.: Some unsolved problems. In: Combinatorics, Geometry and Probability, Cambridge, 1993, pp. 1-10. Cambridge University Press, Cambridge (1997)

13. Erdôs, P., Hickerson, D., Pach, J.: A problem of Leo Moser about repeated distances on the sphere. Am. Math. Mon. 96, 569-575 (1989)

14. Erdős, P., Pach, J.: Variations on the theme of repeated distances. Combinatorica 10, 261-269 (1990)

15. Erdős, P., Stone, A.H.: On the structure of linear graphs. Bull. Am. Math. Soc. 52, 1087-1091 (1946)

16. Füredi, Z., Simonovits, M.: Triple systems not containing a Fano configuration. Comb. Probab. Comput. 14, 467-484 (2005)

17. Grünbaum, B.: A proof of Vázsonyi's conjecture. Bull. Res. Counc. Isr. Sect. A 6, 77-78 (1956)

18. Heppes, A.: Beweis einer Vermutung von A. Vázsonyi. Acta Math. Acad. Sci. Hung. 7, 463-466 (1957)

19. Hopf, H., Pannwitz, E.: Aufgabe Nr. 167. Jahresber. Dtsch. Math.-Verein. 43, 114 (1934)

20. Keevash, P., Sudakov, B.: The Turán number of the Fano plane. Combinatorica 25, 561-574 (2005)

21. Kupitz, Y.S., Martini, H., Wegner, B.: Diameter graphs and full equi-intersectors in classical geometries. In: IV International Conference in "Stochastic Geometry, Convex Bodies, Empirical Measures \& Applications to Engineering Science," vol. II, Tropea, 2001. Rend. Circ. Mat. Palermo (2) Suppl. No. 70, part II, pp. 65-74 (2002) 
22. Martini, H., Soltan, V.: Antipodality properties of finite sets in Euclidean space. Discrete Math. 290, 221-228 (2005)

23. Mubayi, D., Pikhurko, O.: A new generalization of Mantel's theorem to $k$-graphs. J. Comb. Theory (B) 97, 669-678 (2007)

24. Neaderhouser, C.C., Purdy, G.B.: On finite sets in $E^{k}$ in which the diameter is frequently achieved. Period. Math. Hung. 13, 253-257 (1982)

25. Pach, J., Agarwal, P.K.: Combinatorial Geometry. Wiley, New York (1995)

26. Simonovits, M.: A method for solving extremal problems in graph theory. Stability Problems. In: Erdôs, P., Katona, G. (eds.) Theory of Graphs, pp. 279-319. Academic Press, New York (1968)

27. Spencer, J., Szemerédi, E., Trotter, W., Jr.: Unit distances in the Euclidean plane. In: Graph theory and Combinatorics, Cambridge, 1983, pp. 293-303. Academic Press, London (1984)

28. Straszewicz, S.: Sur un problème géométrique de P. Erdős. Bull. Acad. Pol. Sci. Cl. III. 5, 39-40 (1957)

29. Sutherland, J.W.: Lösung der Aufgabe 167. Jahresber. Dtsch. Math.-Verein. 45, 33-35 (1935)

30. Swanepoel, K.J.: A new proof of Vázsonyi's conjecture. J. Comb. Theory Ser. A doi: 10.1016/ j.jcta.2007.08.006 (in press)

31. Swanepoel, K.J., Valtr, P.: The unit distance problem on spheres. In: Pach, J. (ed.) Towards a Theory of Geometric Graphs. Contemporary Mathematics, vol. 342, pp. 273-279. American Mathematical Society, Providence (2004)

32. Székely, L.A.: Crossing numbers and hard Erdős problems in discrete geometry. Comb. Probab. Comput. 6, 353-358 (1997)

33. van Wamelen, P.: The maximum number of unit distances among $n$ points in dimension four. Beitr. Algebra Geom. 40, 475-477 (1999) 